\title{
Temperature Nanotracers for Fractured Reservoirs Characterization
}

\author{
Mohammed Alaskar ${ }^{\mathrm{a}}$, Morgan Ames ${ }^{\mathrm{b}}$, Chong Liu ${ }^{\mathrm{c}},{\mathrm{Kewen} \mathrm{Li}^{\mathrm{b}} \text {, and Roland Horne }}^{\mathrm{b}}$ \\ ${ }^{a}$ EXPEC Advance Research Center, Saudi Aramco, Core Area, Saudi Aramco, Dhahran, Saudi Arabia \\ ${ }^{\mathrm{b}}$ Department of Energy Resources Engineering, Stanford University, 367 Panama Street, Stanford, CA 94305, USA \\ ${ }^{c}$ Department of Materials Science and Engineering, Stanford University, 476 Lomita Mall, Stanford, CA 94305 \\ e-mail: askarmn@aramco.com; mames@stanford.edu; chong813@stanford.edu; kewenli@stanford.edu; \\ horne@stanford.edu
}

Keywords: nanoparticle, silica, deoxyribonucleic acid (DNA), thermochromic, inverse problem

\begin{abstract}
Nanoparticle tracers are being investigated as a potential tool to measure temperature distributions in subsurface reservoirs. If the temperature distributions could be measured more precisely, this would greatly enhance the ability to estimate other reservoir properties, which would in turn inform reservoir engineering and field management decisions. Work toward two distinct but parallel objectives are described here. The first is to rank the informativity of various tracer candidates using modeling. The second is to develop temperature sensitive tracers experimentally, such that they would be capable of characterizing fracture properties and temperature distribution.
\end{abstract}

The design of temperature-sensitive tracers is built around the design of the temperature sensing mechanism. In other words, the mechanism by which temperature is measured and the form and resolution of the resulting data, or response, have a profound impact on how informative that tracer can be about thermal breakthrough. Therefore, it is important to model the responses of candidate tracers in the context of an inverse problem to determine their relative informativity. In order to quantify tracer informativity for the simplified case in which the reservoir consists of a single parallel-plate fracture, one-dimensional analytical models were used to calculate the responses of four tracer types: conservative solute tracer, dye-releasing nanotracer, threshold nanoreactor, and temperature-time tracer (TTT). Inverse modeling was performed for various scenarios, and thermal breakthrough curves were calculated for each solution found. Tracers investigated in this study were found to perform very well, with small ranges of uncertainty for both thermal breakthrough forecasting and fracture property estimation. This was because the problem was highly constrained by defining the reservoir as a single fracture. For more realistic reservoir models consisting of complex fracture networks, it is expected that the uncertainty ranges would be larger and that all three temperature-sensitive tracers investigated here will outperform conservative solute tracers because their responses contain information about the temperature distribution.

Experiments were performed to evaluate several potential nanosensor candidates for their temperature-sensitivity and to investigate particle mobility through porous and fractured media. Temperature-sensitive particles investigated in this study include the 1 
Alaskar et al.

irreversible thermochromic, dye-attached silica and silica-protected DNA particles. A combined heat and flow test confirmed the temperature-sensitivity of the irreversible thermochromic particles by observing the color change. A detectable change in the fluorescent emission spectrum of the dye-attached silica particles upon heating was observed.

The processing and detection of silica-encapsulated DNA particles with hydrofluoric acid chemistry was tested. A protocol to release the DNA by dissolving the silica layer without completely destroying the DNA was established. The silica-encapsulated DNA particles were flowed through a porous medium at high temperature. Some dissolution of silica particles was observed, leading to a reduction in their size.

This research showed that synthesizing particles to respond to a specific reservoir property such as temperature is feasible. Using particles to measure reservoir properties is advantageous because particles can be transported to areas in the reservoir that would not be accessible by other means and therefore provide measurements deep within the formation.

\section{INTRODUCTION}

In both geothermal and oil environments, reservoir description including fracture characterization plays an important role in new field development and existing asset management. Despite advances in seismic, tracer testing and other imaging and sensing technologies, reservoirs (particularly fracture networks) are still poorly known. The flow through natural and engineered geothermal reservoirs as well as through carbonate rock formations is largely fracture-dominated. This has spurred a wide interest among researchers in developing new methods to characterize reservoirs specifically with respect to fracture networks.

Using nanomaterials as reactive tracers could enhance control of their response to temperature, potentially providing more information about the reservoir temperature distribution, fracture geometry, and fracture network topology. Nanotechnology provides promising possibilities for the creation of threshold reactive tracers. Redden et al. (2010) suggested the use of encapsulated nanoreactors to infer the temperature history in geothermal reservoir applications. Encapsulating the reactants in a nanoparticle or nanoreactor could provide more control over the conditions under which reaction can proceed. Particle tracers that release dye upon reaching a threshold temperature are another possibility (Alaskar et al., 2011). Williams et al. (2010) also proposed a dye-release mechanism, in which encapsulated dyes are released once a threshold temperature is reached. Rose et al. (2011) proposed surface-modified quantum dots to measure the fracture surface area at the interwell spacing in enhanced geothermal systems. Saggaf (2008) envisioned futuristic multifunctional nanorobots (called resbots) to measure and store information about pressure, temperature and fluid type. Temperature-sensitive nanomaterials have already been used in the biomedical industry for drug delivery to particular types of body cell that differ in temperature from normal cells (Sutton et al., 2007). Nanoparticle drug carriers are made to target disease-specific locations and release the drug at controlled rates (Kreuter, 1994; Moghimi et al., 2001; Panyam and Labhasetwar, 2003; Panyam et al., 2003). Thermally-activated nanoparticles in biomedical applications were designed to respond to temperatures slightly above $37^{\circ} \mathrm{C}$ (Chilkoti et al., 2002). Although this temperature is much lower than that in subsurface reservoirs, this concept shows promise. Thus, synthesizing temperature-sensitive 
nanomaterials that are applicable for subsurface reservoirs may be feasible. Because such sensing mechanisms are central to the design of these tracers, it is important to know which scheme would provide the most useful information.

The goal in making temperature-sensitive tracers is to make a tool that is capable of providing the best possible information about future reservoir behavior. Therefore, the central design attributes for such tracers is their informativity and the type of information they provide. There are other very significant attributes, such as the cost of manufacturing and the level of technical challenge, but these are secondary to the informativity. For example, if a tracer that can measure the highest temperature it encountered in the reservoir is cheap to make and technically easy to implement in a tracer test, but the information it provides cannot be used to reliably forecast future reservoir behavior, then it is not a good tracer candidate. Therefore, it is important to know how informative a tracer is with respect to future behavior prior to designing its specific attributes such as material and structure. Modeling can provide such insight, which can in turn be used to help direct effort and resources toward the most robust tracer with respect to informativity, level of technical challenge, and cost.

The work described here was performed with two distinct but complementary goals in mind. The first was to model the responses of tracer candidates and quantitatively rank their informativity for characterizing fracture networks in the reservoirs and forecasting their future behavior. Simple inverse modeling was performed for several promising tracer candidates and a reservoir model consisting of a single fracture. The second goal was to develop nanotracers experimentally capable of acquiring specific data about the reservoir temperature near the wellbore and far out in the formation and correlate such information to fracture geometry and fracture network topology. Experiments were used to investigate a number of temperature-sensitive nanoparticle candidates that have a reaction temperature within a temperature range appropriate to geothermal and oil reservoirs.

\section{TEMPERATURE-SENSITIVE NANOTRACER MODELING}

It is critical in the design of temperature-sensitive tracers to rank candidate tracers by their informativity. To this end, analytical models were used to calculate responses for four types of tracers flowing in a single fracture: traditional conservative solute tracer (CSTs), dye-releasing nanotracer (DRNTs), threshold nanoreactors (TNRs), and temperature-time tracers (TTTs). The reservoir temperature distribution was modeled using the analytical solution for a single fracture. Inverse analysis was carried out for this simple problem, and an ensemble of 1000 solutions was obtained for each tracer candidate.

\subsection{Modeling Tracer Responses}

Return curves for all tracers were calculated using the one-dimensional analytical solution to the advection-dispersion equation with flux initial and boundary conditions (Kreft and Zuber 1978), which is given in Equation 1.

$$
C(x, t)=m x / q \sqrt{4 \pi D_{\text {Taylor }} t^{3}} \cdot \exp \left(-(x-u t)^{2} / 4 D_{\text {Taylor }} t\right)
$$


Alaskar et al.

where $C, x, t, m, q, D_{\text {Taylor }}$, and $u$ are tracer concentration, position, time after initial tracer injection, tracer mass injected, volumetric injection flowrate, Taylor dispersion coefficient, and average fluid velocity, respectively.

All temperature distributions used to model tracer responses were calculated using the analytical solution for temperature in a single fracture derived by Gringarten and Sauty (1975). When the flow channel has a porosity of $100 \%$, this solution reduces to the parallel plate solution, which is given in Equation 2.

$$
T(x, t)-T_{0} / T_{I N J}-T_{0}=\operatorname{erfc}\left[\left(x / 2 b u \rho_{W} C_{W}\right) \cdot\left(t-(x / u) / K_{R} \rho_{R} C_{R}\right)^{-0.5}\right]
$$

where $T, T_{0}, T_{I N J}, b, \rho_{W}, C_{W}, K_{R}, \rho_{R}$, and $C_{R}$ are reservoir temperature, initial reservoir temperature, injection temperature, fracture half-aperture, water density, water specific heat capacity, rock thermal conductivity, rock density, and rock specific heat capacity, respectively, and all other parameters are previously defined.

Random Gaussian noise was added to all tracer responses to create the synthetic datasets to be fit in the inverse analysis.

\subsubsection{Conservative Solute Tracers}

Conservative solutes such as fluorescein and naphthalene sulfonates are used commonly as tracers to characterize reservoirs. These tracers are very useful for characterizing well connectivity, but lack the capability to measure fracture properties or reservoir temperature distribution. Therefore, these tracers are expected to represent the lower bound on tracer informativity, which is useful to model in order to establish a baseline for comparison. The dispersion coefficient was modeled using the analytical solution for the Taylor dispersion coefficient in a parallel-plate fracture given by James and Chrysikopoulos (2003). This solution is shown in Equation 3.

$$
D_{\text {Taylor, sol }}=\mathfrak{D}_{\text {Sol }}+\left(2 u^{2} b^{2} / 105 \mathfrak{D}_{\text {Sol }}\right)
$$

where $D_{\text {Taylor, sol }}$ and $\mathfrak{D}_{\text {Sol }}$ are the solute Taylor dispersion coefficient and solute diffusivity, respectively, and all other parameters are previously defined.

The modeled tracer response for a conservative solute tracer and the corresponding synthetic dataset are illustrated in Figure 1.

\section{Figure 1}

\subsubsection{Dye Releasing Nanotracers}

One promising nanotracer candidate under investigation is a nanoparticle tracer with dye attached to the particle surface which desorbs when a threshold temperature is reached. This has the potential to provide useful information about the reservoir location at which the dye was released because there are fundamental differences in the transport properties of solutes and nanoparticles. Because nanoparticles are typically at least two orders of magnitude larger than solute molecules, their diffusivities are correspondingly smaller, as described by the Stokes-Einstein equation (Equation 4). 


$$
\mathfrak{D}_{N P}=k_{B} T_{A B S} / 3 \pi \mu d_{P}
$$

where $\mathfrak{D}_{N P}, k_{B}, T_{A B S}, \mu$, and $d_{P}$ are nanoparticle diffusivity, Boltzmann's constant, absolute temperature, fluid viscosity, and nanoparticle diameter, respectively.

Particles have low diffusivities and due to their size exclusion from walls and small aperture regions, they tend to stay in the faster fluid streamlines, often resulting in earlier breakthrough and lower field-scale dispersivities than solutes (Reimus 1995). A competing effect of the low diffusivity is that the velocity gradient across the fracture spreads the particle front more readily than the solute front, leading to a larger Taylor dispersion coefficient. Becker et al. (1999) performed a tracer test at a fractured granite field site in which both solute and neutrally-buoyant polystyrene particles were injected. They observed that the particles achieved breakthrough $10 \%$ earlier than the solute, and thus estimated that the particles were excluded from $10 \%$ of the fracture volume. They dismissed matrix diffusion as a possible reason for the difference in breakthrough times because the matrix rock was very impermeable. James and Chrysikopoulos (2003) derived a closed form analytical solution for the Taylor dispersion coefficient and effective average particle velocity accounting for the exclusion of particles from slower streamlines due to their finite size (Equations 5 and 6, respectively). This size exclusion phenomenon effectively causes the average particle velocity to increase and the overall dispersion to decrease compared to the theoretical case where they experience no size exclusion.

$$
D_{\text {Taylor, } N P}=\mathfrak{D}_{N P}+\left(2 u^{2} b^{2} / 105 \mathfrak{D}_{N P}\right) \cdot(1-(e / 2 b))^{6}
$$

$$
u_{e f f, N P}=u\left(1+(e / 2 b)-0.5(e / 2 b)^{2}\right)
$$

where $D_{\text {Taylor, } N P}, \mathfrak{D}_{N P}, e$, and $u_{e f f, N P}$ are the nanoparticle Taylor dispersion coefficient, the nanoparticle diffusivity, the size in the aperture dimension of the fracture from which nanoparticles are excluded, and the effective average velocity of nanoparticles, respectively, and all other parameters are previously defined. It can be seen that Equation 5 is equivalent to Equation 3 modified by the term containing the exclusion parameter $e$. Increasing $e$ causes particle dispersion to decrease. Similarly, the effective average particle velocity in Equation 6 is equivalent to the average fluid velocity $u$ modified by a term containing the exclusion parameter $e$. Increasing $e$ causes the effective average particle velocity to increase. James and Chrysikopoulos (2003) assumed that the particles were excluded from a region the size of the particle diameter in the dimension of the fracture aperture (i.e. $\left.e=d_{P}\right)$. In this work, the exclusion from $10 \%$ of the fracture aperture estimated by Becker et al. (1999) was used instead in order to reflect field observations that particles were excluded from $10 \%$ of the fracture volume (i.e. $e=0.2 b$ ).

The utility of a dye-releasing nanotracer lies in the response of the released dye, which traveled some distance with transport properties of a particle before desorbing when encountering the threshold temperature and traveling the remaining distance with the transport properties of a solute. Thus, if the return curve of the released dye is very similar to that of the nanoparticles, then it traveled mostly as a particle, indicating that the reservoir is in a late stage of cooling. Conversely, if the return curve is very similar to that of a simultaneously injected conservative tracer with the same transport properties, then it traveled mostly as a solute, 
Alaskar et al.

indicating that the reservoir is in an early stage of cooling. The return curve of the released dye was modeled as the convolution of the nanoparticle return curve at the location that the dye is released (i.e. the first encountered location where the reservoir temperature equals the threshold temperature) and the return curve for solute traveling the remaining distance (Equations 7 and 8).

$$
\begin{gathered}
C_{R D}(t)=\int_{0}^{t} \frac{m x_{R}}{q \sqrt{4 \pi D_{\text {Taylor, NP }}(t-\tau)^{3}}} \exp \left(-\frac{\left(x_{R}-u_{e f f, N P}(t-\tau)\right)^{2}}{4 D_{\text {Taylor, NP }}(t-\tau)}\right) \cdot \frac{\left(L-x_{R}\right)}{\sqrt{4 \pi D_{\text {Taylor, Sol }} \tau^{3}}} \exp \left(-\frac{\left(L-x_{R}-u \tau\right)^{2}}{4 D_{\text {Taylor, Sol }} \tau}\right) \partial \tau \\
x_{R}=\min x \text { where } T(x)=T_{T H R E S H}
\end{gathered}
$$

where $C_{R D}(t), \tau, x_{R}, L$, and $T_{T H R E S H}$ are concentration of released dye measured at the production well, time integration variable, and threshold temperature, respectively, and all other parameters are previously defined.

The tracer responses calculated for an intermediate cooling stage are illustrated in Figure 2.

\section{Figure 2}

\subsubsection{Threshold Nanoreactors}

Another promising candidate being investigated is the threshold nanoreactor. Redden et al. (2010) suggested the use of encapsulated nanoreactors to infer the temperature history in geothermal reservoir applications. Encapsulating the reactants in a nanoparticle or nanoreactor could provide more control over the conditions under which reaction can proceed, such as imposing a temperature threshold below which reaction does not occur. Potentially, one could use the response for threshold nanoreactors in tandem with the response for another reactive tracer with the same transport and reaction properties but no temperature threshold to infer details of the temperature distribution. Such a tracer test was modeled using the analytical solutions for concentrations of both encapsulated compounds (parent and daughter) derived by Ames et al. (2013), given in Equations 9-12.

$$
\begin{gathered}
C_{A, D}(t)=\frac{C_{A}}{C_{A 0}} \frac{C_{N P}}{C_{M A X}}=\frac{m x / C_{M A X}}{q \sqrt{4 \pi D t^{3}}} \exp \left(-\left(\int_{0}^{t} k(\tau) \partial \tau+\frac{(x-u t)^{2}}{4 D t}\right)\right) \\
k(\tau)=A \exp \left(-E_{A} / R T_{A B S}(\tau)-\right) \\
\tau=x / u \\
C_{B, D}=C_{N P} / C_{M A X}-C_{A, D}
\end{gathered}
$$

where $C_{A, D}(t), C_{N P}, k(\tau), A, E_{A}, R, T_{A B S}(\tau)$, and $C_{B, D}$ are dimensionless concentration of reactant within particles collected at the production well, concentration of nanoparticles at the production well, reaction rate constant, Arrhenius pre-exponential factor, reaction activation energy, gas constant, absolute temperature encountered by the particles as a function of time (which is equivalent to the temperature distribution in the fracture), and dimensionless concentration of daughter product within particles collected at the production well, respectively, and all other parameters are previously defined.

The calculated responses are illustrated in Figure 3. 


\section{Figure 3}

\subsubsection{Temperature-Time Tracers}

Determining the upper and lower bounds of informativity is a valuable exercise that provides reference points for comparison with other tracers. As mentioned in Section 2.1.1, conservative solute tracers represent the lower bound on informativity. The tracers described in this section represent the upper bound. The most informative tracer possible is one that is capable of measuring and recording detailed temperature-time histories encountered by the tracer particles in the subsurface. A tracer that could report physical locations visited by the particles in real time would be a more informative tracer from a reservoir characterization standpoint, but Global Positioning System (GPS) technology is clearly not feasible for this application. Because specific locations and dimensions of the fractures controlling flow cannot be measured directly, time must be used as a proxy for location in order to characterize the specific fracture geometry in the subsurface. In order to record entire temperature-time histories in detail and in a form that can be interpreted, a tracer would require several components: a temperature sensor for continuous temperature measurement, a timer, and memory. This ideal tracer will be referred to henceforth as a Temperature-Time Tracer (TTT).

The responses of TTTs consist of both the particle return curve and the temperature-time response. The former was calculated using Equations 1, 4, 5, and 6. The latter was calculated using Equation 2 with a fixed operation time and modified to transform the $x$ coordinate to a travel time coordinate (Equation 11). The calculated responses are illustrated in Figure 4.

\section{Figure 4}

\subsection{Single Fracture Inverse Problem}

Inverse analysis was carried out in which the synthetic data for each type of tracer was fit by adjusting fracture parameters width $W$ and half-aperture $b$ using the sequential quadratic programming optimization method. The physical and operational parameters used in all cases are summarized in Table 1.

Table 1

As shown in Table 1, the true solution used to generate the synthetic data is $W=1000 \mathrm{~m}$ and $b=1 \mathrm{~mm}$. The fracture length $L$ of $1000 \mathrm{~m}$ was assumed to be known. The projected thermal breakthrough curves corresponding to each solution and that of the true solution were calculated using Equation 2 with the $x$ coordinate fixed to equal $L$.

\subsubsection{Sensitivity Analysis}

A sensitivity analysis was carried out to determine how sensitive the ensemble of solutions is to operation time (i.e. cooling stage), threshold temperature, and amount of noise in the data.

All of these parameters affect how useful each candidate would be. The parameter values used in this sensitivity analysis are summarized in Table 2 .

\section{Table 2}


Alaskar et al.

An ensemble of 1000 solutions was obtained for each of the six cases and for all tracer candidates, except for cases which are not applicable to certain candidates. That is, there is no threshold temperature for the conservative solute tracer and the TTT, and operation time does not affect the conservative solute tracer response, so these cases were not modeled.

\subsubsection{Inverse Problem Results}

The thermal breakthrough curves corresponding to the ensemble of 1000 solutions corresponding to each sensitivity case are illustrated for the conservative solute tracer, dye-releasing nanotracer, threshold nanoreactor, and TTT in Figures 5-8, respectively.

\section{Figure 5}

\section{Figure 6}

\section{Figure 7}

\section{Figure 8}

The TTTs performed better than the other tracers investigated in this study at projecting thermal breakthrough, because the problem was constrained to be a single rectangular fracture of known length with no flow in the matrix and known, homogeneous thermal properties of rock. In a more realistic inverse problem, the uncertainty would be considerably larger.. However, this simple problem was used to determine if there were any noticeable differences between the inverse problems for each tracer on the most basic level. If any of the tracers had not performed well for such a simple, constrained problem, then it would have certainly failed in a more complex case.

The range of decision variables estimated in the inverse analysis are shown for selected cases in Figure 9.

\section{Figure 9}

Estimates of decision variables (i.e. fracture parameters) for all tracers were roughly within $4 \%$ of values for fracture length and $5 \%$ for fracture aperture (disregarding outliers), with TTTs and conservative solute performing slightly better than dye release and nanoreactors. However, all tracers were found to be informative about thermal breakthrough for the single fracture problem, with no candidate standing out from the rest. These solutions lay along a curve (or equivalently, a trough in the objective function surface) because of the nonuniqueness of the problem. All tracers showed some sensitivity to noisy data, reflected by increasing uncertainty and inaccuracy in thermal breakthrough forecasts and fracture parameter estimation. All tracers seemed relatively insensitive to operation time and threshold temperature (when applicable). For more realistic reservoir models consisting of complex fracture networks, it is expected that all three temperature sensitive tracers would outperform conservative solute tracers because their responses would contain information about the temperature distribution.

\section{TEMPERATURE-SENSITIVE NANOTRACER EXPERIMENTS}

In this study, we investigated the temperature-sensitivity of nanosensors that undergo detectable and irreversible changes at a specific temperature. In our previous work (Alaskar et al. 2010, 2011, 2012, 2013 and 2014), we have described the investigation of 
the transport of particles of different materials, physical and chemical properties and flowed through various flow media such as sand or glass bead packed tubes, porous and fractured cores and at microscale using silicon micromodels. Here, we describe the preliminary testing that was carried out for three potential temperature-sensitive nanoparticles; namely, fluorescent dye-attached silica, thermochromic polymer and silica-encapsulated DNA particles. A combined heat and flow test was performed for the thermochromic polymer and silica-encapsulated DNA particles only. The fluorescent dye-attached silica nanoparticles were tested for their temperature sensitivity without simultaneously being flowed through porous medium.

In the following sections, the heat and/or transport tests of the temperature nanosensor candidates will be discussed.

\subsection{Fluorescent Dye-attached Silica Temperature Nanosensors}

As silica particles have been proven to have transported through sandstone core successfully, we changed their surface properties to explore their temperature response. According to $\mathrm{Wu}$ et al. (2008), when free fluorescent dye molecules were attached to the surface of silica nanoparticles, through energy transfer, the fluorescent properties of these molecules were changed. When the covalent bond between fluorescent dye molecule and surface-modified silica nanoparticle is broken under high temperature, the difference of fluorescent behavior before and after heating experiment could be detected. The characteristic of the temperature measurement is an observable change in the fluorescent emission spectrum of the attached dye before and after the exposure to heat.

\subsubsection{Synthesis of Fluorescent Dye-attached Silica Nanoparticles}

Nanoparticle synthesis and dye attachment followed the protocol suggested by Saleh et al. (2010). A schematic representation of silica nanoparticle surface modification and dye attachment is shown in Figure 10. First, silica nanoparticles (Nanogiant, LLC) were modified by attaching an amino group to their surfaces. In a typical reaction, $0.5 \mathrm{ml}$ of 3-Aminopropyltriethoxysilane (APTS) was added to $100 \mathrm{mg}$ silica nanoparticle suspended in $25 \mathrm{ml}$ of toluene under nitrogen and heating to $\sim 95^{\circ} \mathrm{C}$ for 4 hours. The resulting particles were washed by centrifugation in ethanol and acetone (10 $\mathrm{min}$ at 4,400 rpm). Then the particles were dried overnight at $\sim 95^{\circ} \mathrm{C}$. The particles were about $350 \mathrm{~nm}$ in diameter. Second, the dye molecule (Oregon 488, Invitrogen) was attached to the modified silica particle surface. A suspension of $1.0 \mathrm{mg}$ of the amino-modified silica nanoparticles in a mixture of $1 \mathrm{ml}$ of ethanol and $15 \mu 1$ of a $10 \mathrm{mmol} / \mathrm{l}$ phosphate buffer $(\mathrm{pH} 7.3)$ was reacted with $12.7 \mu 1$ of dye molecule solution $(1 \mathrm{mg} / \mathrm{ml} \mathrm{water}$ solution) in dark for 3 hours at room temperature. The resulting particles were washed by centrifugation (10 min at 4,400 rpm) in ethanol and acetone.

Surface modification and dye attachment reaction was also performed on a monolayer of silica nanoparticles on quartz substrate using the same experimental parameters. In other words, two fluorescent dye-attached silica particles samples were prepared; one sample where particles were suspended in solution (water) and a second sample in which particles were deposited on quartz substrate. 
Alaskar et al.

\subsubsection{Characterization of Fluorescent Dye-attached Silica Nanoparticles}

Fluorescent microscopy and fluorescent spectroscopy were used to characterize and verify the dye attachment to the silica nanoparticles. Fluorescent microscopy characterization, as shown in Figure 11, was conducted using the substrate base dye-attached silica nanoparticle sample. The whole substrate was fluorescing (some spots were brighter because more fluorescent molecules were being attached, suggesting a successful attachment of the dye to the silica nanoparticles.

The fluorescence spectra of free dye molecule solution, silica nanoparticle suspension, and dye-attached silica nanoparticles in solution and on substrate (prior to heating) were measured as shown in Figure 12. All samples were excited at wavelength of 400 $\mathrm{nm}$. The Oregon 488 dye has its maximum emission peak at wavelength of $530 \mathrm{~nm}$ (Figure 12a). The fluorescent spectrum of bare silica nanoparticles (no dye-attachment) was acquired as a control and found to have no fluorescent response. After the dyeattachment, on the other hand, two emission peaks were observed at wavelengths of 425 and $530 \mathrm{~nm}$ (Figure 12b).

\section{Figure 10}

\section{Figure 11}

\section{Figure 12}

\subsubsection{Heating Experiment of Fluorescent Dye-attached Silica Nanoparticles}

A heating experiment was conducted using the fluorescent dye-attached silica nanoparticles that were deposited on quartz substrate. The substrate was placed on a hot-plate at a temperature of $200^{\circ} \mathrm{C}$ for $15 \mathrm{~min}$. Then substrate was soaked and washed in ethanol and acetone and then was left to dry in air. Figure 13 shows the fluorescent spectra of the fluorescent dye-attached silica sample prior and after the heat test. An excitation wavelength of $360 \mathrm{~nm}$ was used for both samples. It was observed that the heated sample had a wide peak at $425 \mathrm{~nm}$ that was very different than that before heating. Besides that, shoulder peaks at $380 \mathrm{~nm}$ and $475 \mathrm{~nm}$ and a peak at the spectrum tail $(575 \mathrm{~nm})$ were also observed for both samples. The observable change in the emission peak between the heated and unheated sample could be used as a characteristic of temperature measurement. Therefore, the fluorescent dye-attached silica nanoparticles could potentially be used as a temperature nanosensor for subsurface reservoirs.

\section{Figure 13}

\subsection{Irreversible-color-change Thermochromic Particles}

Chromogenic materials undergo a change in their color in response to electrical, optical or thermal stimulus (Seeboth et al., 2007). Colored chromogen compound that contains chromophore atoms are responsible for the visible color change in chromogenic materials. The color change arises when the visible light is transmitted or reflected by the chromogen (Bamfield, 2001). Among the different categories of chromogenic materials, thermochromics play an important role in a wide range of applications (Seeboth et al., 2007).

Thermochromic materials change their color due to change in temperature, a phenomena known as thermochromism (Bamfield, 2001). There are two primary types of thermochromics, liquid crystals and leuco dyes. Liquid crystals change color based on 
specific light wavelength reflection from their structures. The most common commercial applications include forehead thermometers, fish-tank thermometers and stress testers (White and LeBlanc, 1999). Liquid crystals are used for precise temperature measurement and are difficult to work with as they require specialized manufacturing techniques. Leuco dyes, on the other hand, are easier to synthesize but less accurate than liquid crystals. Leuco dyes, usually organic, involve a mixture of at least two chemical components in order to achieve a thermal response. They are widely used in advertising, consumer packaging, security labels, toys and textiles (White and LeBlanc, 1999). The main shortcoming of the liquid crystals and leuco dyes is the reversibility to original color upon cooling, which hinder them to be useful temperature sensors in subsurface reservoir applications.

Another class of thermochromic materials known as thermochromic permanent change ink is a high temperature activated irreversible thermochromics. These pigments have a wide temperature reaction range ( 60 to $200^{\circ} \mathrm{C}$ ) that may be suitable for oil as well as geothermal applications. Thermochromic inks are manufactured in industrial quantities, which also make them attractive for reservoir application.

The following sections describe the preliminary testing of the temperature sensitivity and transport of irreversible thermochromic particles.

\subsubsection{Irreversible Thermochromic Particles Bench Heat Tests}

Tested samples were acquired from LCR Hallcrest LLC. The irreversible color change ink particles are supplied in two forms: inks and concentrate. The concentrates are used in the formulation of inks using water-based resins. All tests were carried out using the concentrate format.

The pigment concentrates are in the form of ground crystalline solids suspended in water. The suspension concentration was $39 \%$ $( \pm 2 \%)$ solids. The pigment concentrate is slightly alkaline with a $\mathrm{pH}$ in the range of 6-8 at start of printing, and has a density between 1.0-1.1 relative to water. Note that sample specifications were obtained from the manufacturer, based on the product specification and material safety data sheets.

The temperature sensitivity was verified using a bench heat test. Two samples were tested, black and magenta concentrates. The reaction temperature of the black and magenta concentrates was 90 and $60^{\circ} \mathrm{C}$, respectively. These samples of such reaction temperatures were selected to avoid complication in the heat testing due to water boiling. Although these temperatures were below the temperature range of interest, their use served the purpose of investigating the concept of using irreversible thermochromic materials as temperature sensors in subsurface reservoirs.

As specified by the manufacturer, the color density of the thermochromic concentrates should increase with increasing temperature, with distinct color change at the rated temperature and full shade at temperatures of 10 to $20^{\circ} \mathrm{C}$ above the rated temperature. A faint color change is expected to occur at about 10 to $30^{\circ} \mathrm{C}$ below the rated temperature. Therefore, the temperature response was tested at different increments below and above the rated or reaction temperature. The black and magenta concentrates were heated to temperatures ranging from 60 to $100^{\circ} \mathrm{C}$ with $10^{\circ} \mathrm{C}$ increments, and 55 to $135^{\circ} \mathrm{C}$ with increments of $20^{\circ} \mathrm{C}$, respectively. The samples were contained in a 1/8-inch coiled stainless steel tube. The coiled tube was then submerged in an oil bath (Polyscience heat 
Alaskar et al.

circulator model 1147 with working temperature from ambient to $200^{\circ} \mathrm{C}$ ) at desired temperature for three minutes. The heat interval ( 3 minutes) was selected to match the heat test duration performed by the manufacturer on the same samples. Prior to heating, each sample was diluted one part to nine parts of pure water (1:10 dilution). The coiled tube can be seen in Figure 14. The pressure gauge was used to monitor pressure build up for heat tests at or above $100^{\circ} \mathrm{C}$. Before transferring the heated sample into a glass vial, the sample was cooled in air to ambient temperature.

\section{Figure 14}

\section{Figure 15}

\section{Figure 16}

Snapshots of heated samples can be seen in Figure 15. It was observed that the black and magenta samples began to change color at $20^{\circ} \mathrm{C}$ and $5^{\circ} \mathrm{C}$ below the rated temperatures, respectively. The black sample showed a distinct change in color at about $10^{\circ} \mathrm{C}$ above the rated temperature, while the magenta sample reached a fully developed yellow color at $35^{\circ} \mathrm{C}$ higher than the rated temperature. The color intensity was increasing with increasing temperature for both samples. These observations are in qualitative agreement with the manufacturer data. Figure 16 shows the change in color density as function of temperature for various irreversible thermochromic inks. Note these samples were test prints. Heat tests using the permanent thermochromic samples in concentrate format such as those used here are expected to have a degree of variation in color intensities. For example, the magenta color was not fully developed at around the rated temperature $\left(60^{\circ} \mathrm{C}\right)$, although the test print results (Figure 16) indicated that the color density reached a plateau beyond the sample rated temperature. Similarly, the color intensity of the black concentrate was gradually increasing with increasing temperature. The distinct color change occurred at higher than the rated temperature. It needs to be clear that these observations are based on visual comparisons not actual optical density measurements.

It was observed that the particles have irregular shapes and a wide size distribution (Figure 17a). initially. the particles were ground crystalline solids. Upon heating, the melted particles were more spherical in shape with average particle size of $2 \mu \mathrm{m}$ in diameter and smaller. This is evident in the micrograph in Figure 17b.

The bench heat tests clearly confirmed the temperature sensitivity of the irreversible thermochromic concentrates. Because these particles will be flowing through the reservoir rock pore spaces, it was essential to test their temperature sensitivity while transported through a porous medium. Thus a combined heat and transport test was conducted. The magenta particle suspension was transported through a column packed with glass beads. The details of this injection are provided in the next section.

\section{Figure 17}

\subsubsection{Magenta Thermochromic Particles Combined Heat and Transport Experiment}

The objective of the combined heat and transport experiment was to test the thermochromic concentrate temperature sensitivity during its flow through a porous medium. The experimental apparatus used during the transport experiment of the magenta thermochromic sample can be seen in Figure 18. The apparatus consists of two water pumps, Dynamax (Model SD-200, head 50 
WSS) and Cole-Parmer Masterflex (Model 7518-10), manometer, heating tape (BriskHeat), thermocouple and thermocouple signal conditioner (Digi-Sense, Model 92800-00), water vessel and glass column (Condenser, Liebig 24/40, No.2360-300). The glass condenser was used because it allows for visual observation of the color change under heat. Because this is a glass model, it is only applicable for testing thermochromic samples with reaction temperature of $80^{\circ} \mathrm{C}$ and below. Higher temperatures would boil the water under atmospheric pressure. Using back pressure to prevent boiling of water was not possible because the glass tube may not withstand high pressure. Glass beads (mesh 40-45, batch 1784 from Microbeads) were packed inside the inner tube of the condenser. Water was heated using the heating tape in the water vessel and along the circulation loop. The hot water was circulated from the water vessel to the annulus of the condenser using the Cole-Parmer Masterflex pump. The temperature was measured in the annulus, within the glass beads at column outlet and at the water vessel. The temperature signal was read directly using the scanning thermocouple thermometer signal conditioner. The pressure difference between the inlet and outlet of the column was measured using a manometer.

Prior to the transport experiment, the column was saturated with testing fluid (i.e. pure water) by evacuating the column (using Welch vacuum pump) to a vacuum pressure of 50 millitorr for four hours. The pore volume and porosity was determined based on the volume of water displaced through the column. The column water permeability was determined using Darcy's law for vertical flow of single-phase incompressible fluids. The physical properties of the column are summarized in Table 3. The inlet and outlet of the column was fitted with screen mesh (mesh 200) to prevent the glass beads from flowing out. The thermochromic sample was injected through an injection loop using the Dynamax pump from the bottom of the column. The heated zone was about $5 \mathrm{~cm}$ from the inlet of the column (Figure 18b). The injection flow rate was kept constant at $1.0 \mathrm{~cm}^{3} / \mathrm{min}$. The column was flushed with pure water for ten pore volumes prior to the thermochromic sample injection. The thermochromic sample was diluted one to two parts of pure water and the volume injected was approximately $37 \%$ of the pore volume $\left(8 \mathrm{~cm}^{3}\right)$. The particle suspension was thoroughly sonicated prior to the injection using a Branson 2510 Sonicator. One and half pore volumes of pure water were post injected and effluent samples were collected at volumes of $2.5 \mathrm{~cm}^{3}$. The temperature was held constant at $80^{\circ} \mathrm{C}\left(20^{\circ} \mathrm{C}\right.$ above the rated temperature of the magenta sample) because the sample was expected to reach its full color density $20^{\circ} \mathrm{C}$ above the rated temperature. Although this temperature is lower than the temperature range in many subsurface reservoirs, the aim of this experiment was to verify the temperature functionality of the thermochromic sample while flowing through a porous medium.

\section{Figure 18}

\section{Table 3}

Direct observation of the temperature sensitivity as a change in color of the thermochromic sample during its transport through a porous medium was the main focus of this experiment. Visual observation (Figure 19) confirmed unambiguously the temperature sensitivity of the thermochromic sample during its transport through the glass beads. The sample switched color immediately from off-white to magenta at the entry of the heated zone of the column. The injected slug flowed with a clearly apparent sample front as it moved through the column. 
Alaskar et al.

The breakthrough of the particles occurred about 0.93 of the pore volume with a residence time of 20 minutes, slightly faster than the bulk fluid. The progression of the particle transport through the column at different time intervals can be seen in Figure 20 . The bulk of the particles were flushed out of the column by the post-injection of about 1.5 pore volumes of pure water. It was observed that air started to evolve from the water at the entry of the column due to heating, which caused some particles to deposit at the airwater interface (AWI). Particles interact with the AWI in similar ways as they interact with solid surfaces (or grains). The AWI serves as a favorable attachment site for particles due to their hydrophobicity (Keller and Auset, 2007). Wan and Wilson (1994a, 1994b) reported that particles sorbed irreversibly at the AWI. They also observed that particle retention increased with increasing air saturation. The entrapment of the thermochromic particles at the AWI can be seen visually the snapshots (3-10) of Figure 20 . Particles were deposited permanently and could not be remobilized by flushing at higher flow rates.

\section{Figure 19}

\section{Figure 20}

The intensity of the magenta color increased as the thermochromic particles remain under heat for longer time. This can be observed by comparing snapshots 1 and 3 in Figure 20. Particles in snapshot 3 were exposed to heat about 10 minutes longer than those in snapshot 1 . Change in the color intensity as function of time could be particularly useful to determine the geolocation of the particles. The particles had also reshaped to spheres upon heating. Figure 21 shows a comparison between the heated and unheated thermochromic samples. The change in particle shape could be used as another characteristic of temperature measurement. So irregular (rod-like) particles (Figure 21a) may not impose constraint on the particle transport as they will transform to spheres, because the transport of spherically-shaped particles was facilitated; a conclusion based on our earlier experiments into the transport of silver nanowire and hematite nanorice through porous media. The details of those earlier transport studies can be found in Alaskar et al. (2012).

It was concluded that irreversible thermochromic material has the potential to be used as a temperature sensor to measure the temperature of subsurface reservoirs. However, surface characteristics of thermochromic particles and their interaction with constituents of reservoir rock, fluids and reservoir conditions of pressure, salinity are subjects for further investigations.

\section{Figure 21}

\subsection{Silica-encapsulated DNA Nanoparticles}

Deoxyribonucleic acid (DNA) is a nucleic acid that encodes the genetic organisms. Nucleic acids are known for their exceptional capability to store information, encryption and barcoding, allowing for wide range of industrial applications such as food and consumer goods labeling (Bancroft et al., 2001; Popping, 2002). Nucleic acids, however, degrade greatly when subjected to conditions beyond their biological origin. Paunescu et al. (2013) suggested a simple DNA encapsulation inside silica particles. The encapsulation was accomplished by surface modification of the silica particle with ammonium to give it a net positive charge. A negatively charged DNA was then adsorbed on the silica particle of opposite charge. After neutralizing the remaining negative charge of the DNA, a dense shell of silica was grown around the DNA using TEOS by polycondensation (Paunescu et al., 2013). 
The primary application of the silica-encapsulated DNA particles was barcoding for polymer labeling. The authors tested the protected DNA under conditions similar to those in an actual polymers manufacturing process of elevated temperatures and radical treatments. They reported that the DNA, after being heated to temperatures as high as $200^{\circ} \mathrm{C}$, can still be detected and quantified using real-time polymerase chain reaction (qPCR) technique. The DNA concentration was decreasing to a distinct amount with increasing temperature. This behavior may be used as a sensing mechanism of temperature sensor.

The idea of using silica-encapsulated DNA particles as a temperature sensor was based on the fact that the concentration of the DNA changes with temperature. By using calibration curves, it may be possible to relate the DNA concentration to the reservoir temperature and exposure time at that temperature. Calibration curves can be constructed using laboratory controlled heat tests by heating the silica-protected DNA sample to a range of temperatures for different times.

\section{$\underline{\text { 3.3.1 Silica-DNA Particles }}$}

The silica-protected DNA sample was received from the Institute for Chemical and Bioengineering at Eidgenossische Technische Hochschule (ETH) Zurich. The particle size prior and after the encapsulation of the DNA was measured using scanning electron (SEM) and transmission electron (TEM) microscopy imaging. The particle size distribution was determined by counting at least 50 particles per sample. The mean particle size was found to be approximately $175 \mathrm{~nm}$, of which 10-12.5 nm was the thickness of the silica layer (Paunescu et al., 2013). The original sample concentration was $0.1 \mathrm{mg} / \mathrm{ml}$ (with $1.73 \mu \mathrm{g}$ DNA per $1 \mathrm{mg} \mathrm{SiO}_{2}$ ). One volume of the original sample was diluted with six volumes of pure water. The diluted sample of new concentration of $1.43 \times 10^{-2}$ $\mathrm{mg} / \mathrm{ml}$, corresponding to $5.09 \times 10^{9}$ particle/ml, was used during testing.

\subsubsection{Sample Preparation and DNA Quantification}

A real-time polymerase chain reaction (qPCR), Applied Biosystems StepOnePlus Instrument, was used to quantify the DNA. Prior to the DNA amplification, the DNA was released by means of hydrofluoric acid chemistry as suggested by Paunescu et al. (2013).

Buffered hydrofluoric acid, commonly known as buffered oxide etch (BOE), was used to dissolve the silica layer. The BOE is a mixture of ammonium fluoride $\left(\mathrm{NH}_{4} \mathrm{~F}\right)$ and hydrofluoric acid (HF). The addition of $\mathrm{NH}_{4} \mathrm{~F}$ was to slow down and control the attack rate of $\mathrm{HF}$ on oxides. The BOE solution was prepared as follows. First, $40 \mathrm{~g}$ of $\mathrm{NH}_{4} \mathrm{~F}$ powder was mixed with $60 \mathrm{~g}$ of water to make $40 \%$ solution. The $\mathrm{HH}_{4} \mathrm{~F} /$ water solution was sonicated until the $\mathrm{NH}_{4} \mathrm{~F}$ was fully dissolved (15 minutes) and the mixture became clear. The solution was left to cool. Second, $0.5 \mathrm{ml}$ of $49 \% \mathrm{HF}$ acid was then added to the $40 \% \mathrm{NH}_{4} \mathrm{~F}$ solution to make 200:1 BOE solution with HF concentration of $0.24 \%$. The etching rate of this BOE was about $2.18 \mathrm{~nm} / \mathrm{min}$.

The DNA extraction was performed by adding four volumes $(40 \mu 1)$ of the BOE solution to one volume (10 $\mu 1)$ of the silicaencapsulated DNA particles sample (Paunescu et al., 2013). The time the BOE was allowed to react with the silica-protected DNA particles was calculated based on the BOE etching rate and the thickness of the silica shell (about 4.59-5.73 min).

The reaction was halted by separating the DNA from the BOE solution using GET clean DNA spin column kit, (catalog number 786-356/357) obtained from G-Biosciences Geno Technology Inc. The spin columns were supplied with binding buffer (Guanidine 
Alaskar et al.

Hydrochloride), DNA wash (various salts) and TE buffer (Tris and Ethylenediaminetetraacetic acid solution). The separation process was conducted according to the following protocol suggested by the manufacturer of the spin tubes. First, the silicaprotected DNA sample was mixed with the BOE solution (total volume was $50 \mu \mathrm{l}$ ) and allowed to react for the specified time. 250 $\mu 1$ of the binding buffer was added and mixed with the DNA sample. The mixture was transferred to the spin column and centrifuged to bind the DNA, using Eppendorf Microcentrifuge Model 5430, for one minute at 14,000g. The flow-through was discarded and the column was placed back into the same collection tube. $750 \mu 1$ of the DNA wash (cooled on ice before use) was added to the column and centrifuged for one minute at 14,000g. The flow-through was discarded and the column was centrifuged again for one minute at $14,000 \mathrm{~g}$ to ensure that the DNA wash was removed completely. The column was then placed in a clean collection tube (microtube). $50 \mu \mathrm{l}$ of TE buffer (preheated to $55^{\circ} \mathrm{C}$ ) was added to the center of the column membrane and incubated at room temperature for one minute. The column was centrifuged for one minute at $14,000 \mathrm{~g}$. The DNA in the collected sample was then amplified using qPCR.

PCR runs were conducted using the same primers, master mix and primer mix as outlined in Paunescu et al. (2013). Every sample was mixed with $10 \mu 1$ of qPCR master mix (SYBR Green I), $7 \mu 1$ of PCR grade water and $2 \mu 1$ of forward and reverse primer mix. Dilutions of standard amplicon of known concentrations $\left(10^{-8}\right.$ to $\left.10^{-4} \mu \mathrm{g} / \mu \mathrm{l}\right)$ were used to quantify qPCR. All samples were carried out in triplicate.

In order to test the nucleic acid (DNA) release protocol, a test qPCR run to quantify the DNA was carried out using the silicaencapsulated DNA sample at its original concentration. Based on the silica layer thickness and etching rate, the release of the DNA should occur approximately between 4.59-5.73 minutes. Six samples were allowed to react with the BOE solution for specific time before separation using the spin columns. The shortest reaction time used during the qPCR test run was 3 minutes, with an increment of 1 minute for subsequent samples.

The DNA was detected and amplified for all samples. The amplification plot and standard curve for standards and samples can be seen in Figure 22 and 23, respectively. The cycle thresholds $\left(\mathrm{C}_{\mathrm{t}}\right)$, defined as number of cycles required for fluorescent signal to exceed background fluorescent level, showed strong reaction $\left(C_{t} \leq 29\right)$ indicating that there was an abundant amount of target DNA in all samples. There was variability in the amount of DNA among samples ranging from 0.006 to $0.739 \mathrm{pg} / \mathrm{ml}$, with the majority around $0.357 \mathrm{pg} / \mathrm{ml}$.

\section{$\underline{\text { 3.3.3 Silica-encapsulated DNA Combined Heat and Transport Experiment }}$}

A stainless steel (316L) tube packed with sand with inner diameter of $0.46 \mathrm{~cm}$ and length of $30.48 \mathrm{~cm}$ was used. The tube was packed with Ottawa sand with a density of $2.65 \mathrm{~g} / \mathrm{cm}^{3}$ and grain size ranging from 150 to $180 \mu \mathrm{m}$. The sand was washed several times with pure water and dried prior to use. The porosity, pore volume (by resaturation method) and liquid permeability (using Darcy's law for horizontal flow of single-phase incompressible fluid) were measured, and found to be approximately $36.19 \%, 2.0$ $\mathrm{cm}^{3}$ and 9.17 darcy, respectively. 
A schematic of the experimental apparatus used during the combined heat and flow test of the silica-encapsulated DNA particles can be seen in Figure 24. The apparatus consists of a water pump, Eldex (Model PN 597-Optos Pump 2SM, minimum and maximum flow rates of 0.01 and $10 \mathrm{~cm}^{3} / \mathrm{min}$ ), air bath oven manufactured by Blue $\mathrm{M}$ (maximum temperature of $400^{\circ} \mathrm{C}$ ), syringe (Monojet $3 \mathrm{ml}$ ), differential pressure transducer (Validyne Model DP15-56), thermocouple and thermocouple signal conditioner (Digi-Sense, Model 92800-00), balance (Mettler balance Model PE 300), water pocket and a back pressure regulator.

\section{Figure 22}

\section{Figure 23}

\section{Figure 24}

The syringe was used to place the particle suspension through the injection loop. The particle suspension was injected into the sand packed tube at flow rate of $1 \mathrm{~cm}^{3} / \mathrm{min}$ using the water pump. The pressure measurements were collected using a data acquisition card (National Instruments Model USB-6009). The temperature data was recorded directly from the thermocouple signal conditioner. The sand packed tube was placed inside the air bath to control the temperature at which each experiment was conducted. The silica-encapsulated DNA particles were injected at three different temperatures $\left(120,150\right.$ and $\left.180^{\circ} \mathrm{C}\right)$. To prevent water boiling, a back pressure of 4, 7 and 12 atm was provided during the 120,150 and $180^{\circ} \mathrm{C}$ injection experiments using the back pressure regulator. The outflow was cooled down to room temperature before reaching the back pressure regulator using a water jacket. The discharge flow rate was measured using the balance and stop watch to ensure constant steady-state conditions.

The injected volume of the particle suspension was $20 \%$ of the pore volume $\left(2 \mathrm{~cm}^{3}\right)$. The system was flushed with several pore volumes of pure water before and after each injection. The particle suspension injection was carried as follow. First, the air bath oven temperature was raised to desired temperature. A continuous injection of particle-free water was introduced using the water pump. When the inlet and outlet temperatures reached the preset temperature of the air bath, the particle suspension slug was flowed through the porous medium. Typically, four pore volumes of pure water were post-injected and effluent samples were collected at a volume of $0.70 \mathrm{~cm}^{3}$.

After recovery of the silica-protected DNA particles following their flow through the porous medium, it was observed that the silica particles exhibited reduction in their size. Electron micrographs of the silica sample before and after injection can be seen in Figure 25 (a and b). As temperature increased from 25 to $150^{\circ} \mathrm{C}$, the particle size was reduced by about a factor of four.

Analysis of the effluent using electron microscopy showed that particles were aggregating when transported at high temperatures (Figure 25c). This was due to the increased solubility of silica particles and high collision between particles at high temperature (Brinker and Scherer, 1990). Rahman et al. (2007) reported similar observations during their investigation of an optimized sol-gel synthesis of stable silica particles. They observed reduction in the particle size and increased particle aggregation with increasing temperature. 
Alaskar et al.

The silica-protected DNA particles were transported through the sand-packed tube at room temperature as a control experiment. Neither particle size reduction nor aggregation was observed, as seen in the micrographs in Figure 25d, suggesting that the dissolution of silica particles was associated with higher temperature.

\section{Figure 25}

\section{CONCLUSIONS}

Work toward two distinct but parallel objectives has been described here. The first objective is to rank the informativity of various tracer candidates using modeling. The second objective is to develop temperature sensitive tracers capable of characterizing fracture properties and temperature distribution.

In order to quantify tracer informativity for the simplified case in which the reservoir consists of a single parallel-plate fracture, one-dimensional analytical models were used to calculate the responses of four tracer types: conservative solute tracer, dyereleasing nanotracer, threshold nanoreactor, and temperature-time tracer (TTT). The solute tracer and TTT represent the lower and upper bounds on informativity, respectively, and the other two are tracer candidates currently being investigated. Synthetic tracer data was obtained by adding random Gaussian noise to these responses. Inverse modeling was then performed in which the data was fit by adjusting fracture width and half-aperture (with a fixed, known length) to obtain an ensemble of 1000 solutions for each tracer. This analysis was repeated for various operation times, threshold temperatures, and levels of noise in the data in order to identify possible weaknesses associated with a particular type of tracer. Finally, thermal breakthrough curves were calculated for each solution found.

The idealized TTTs performed better than the other tracers at projecting thermal breakthrough, but all tracers did very well because the problem was constrained to be a single fracture of known length with no flow in the matrix and known, homogeneous thermal properties of rock. In a more realistic inverse problem, the uncertainty would be larger. However, this simple problem was used to see if there were any noticeable differences between the inverse problems for each tracer on the most basic level. If any tracer had not performed well for such a simple, constrained problem, then it would have certainly failed in a more complex case. All tracers provided estimates that were roughly within $4 \%$ of fracture length and 5\% of fracture aperture (disregarding outliers), with TTTs and conservative solute performing slightly better than dye release and nanoreactors. However, all tracers were found to be informative about thermal breakthrough for the single fracture problem, with no candidate standing out from the rest. All tracers showed some sensitivity to noisy data, reflected by increasing uncertainty and inaccuracy in thermal breakthrough forecasting and fracture parameter estimation. All tracers seemed relatively insensitive to operation time and threshold temperature (when applicable). For more realistic reservoir models consisting of complex fracture networks to be explored in future work, it is expected that all three temperature sensitive tracers would outperform conservative solute tracers because their responses contain information about the temperature distribution.

The experimental objective is to develop thermally-responsive particles to measure temperature far in the formation and use such measurements to infer other reservoir and fracture properties. This approach involved synthesizing temperature-sensitive particles 
and investigating their mobility through porous and fractured media. This study has shown that temperature-sensitive particles undergo observable change as per their design and can be transported through a reservoir rock.

Several temperature-sensitive particles were investigated, each with a different sensing scheme. These include irreversible thermochromic, fluorescent dye-attached and silica-encapsulated DNA particles. These particles were shown to respond to temperature upon heating.

The irreversible thermochromic particles change their color permanently due to change in temperature. These pigments are available with trigger temperatures as high as $200^{\circ} \mathrm{C}$, and many subsurface reservoirs are within this range. The temperature sensitivity and transport of the irreversible thermochromic particles were tested simultaneously. The thermochromic particles were injected through a heated porous medium. The thermochromic sample changed color during its transport through the heated zone of the porous medium. This confirmed that thermochromic particles can be used to measure the temperature subsurface reservoirs.

In the case of the dye-attached silica particles, the dye was incorporated to the surface of the silica particle by surface modification and dye attachment. The silica particle surface was modified by attaching an amino group, and then the dye molecule was attached to the particle surface by covalent bond. Upon heating, the covalent bond breaks resulting in an observable change in the fluorescent dye emission spectrum. The difference in the fluorescent emission spectrum is the characteristic of the temperature measurement. A heating experiment was conducted on the dye-attached silica particles. It was observed that the fluorescent emission spectrum of the heated sample was very different than that before heating.

The sensing mechanism of the silica-encapsulated DNA particles would be the degradation in the DNA concentration with increasing temperature. The DNA encapsulation was accomplished by functionalizing the surface of the silica particle to yield a positively charged particle. A negatively charged DNA was adsorbed on the positively charged particle. A dense silica layer surrounding the DNA was then deposited using TEOS. At this stage, the DNA is suspended between the core and shell of silica particle. The applicability of the silicate and hydrofluoric acid chemistry with nucleic acid analysis (qPCR) was verified.

The mobility of the silica-encapsulated DNA particles was investigated. Particle suspensions were transported through a sandpacked tube at three different temperatures. Particle size was generally decreased with increasing temperature due to the high rate of dissolution. Also, particles were aggregating with increasing temperature. This was attributed to the increased dissolution and collision between particles at high temperature. On the other hand, reduction in particle size and aggregation were not observed during the particles injection at room temperature. It was concluded that the silica-encased DNA particles should be synthesized with some kind of protective coating to yield particles that are less soluble at high temperature to prevent silica dissolution at these temperature conditions.

Collectively, the results of this study show that carefully designed temperature-sensitive nanomaterials are promising candidates for the analysis of fractured reservoirs. 
Alaskar et al.

\section{ACKNOWLEDGEMENTS}

The authors acknowledge the support of the Department of Energy (under contract number DE-FG36-08GO18192). The authors also thank the Office of the Dean of the School of Earth Sciences at Stanford University for supporting this work. The authors thank LCR Hallcrest for providing the thermochromic samples, and Daniela Paunescu and Robert Grass from the Institute for Chemical and Bioengineering at Eidgenossische Technische Hochschule Zurich, for providing the silica-protected DNA samples.

The authors are grateful to Mohammad Karimi-Fard, Jef Caers, Phil Brodrick, and Obi Isebor for their time and insight.

\section{REFERENCES}

Alaskar, M., Ames, M., Connor, S., Liu, C., Cui, Y., Li, K., and Horne, R. (2012). Nanoparticle and Microparticle Flow in Porous and Fractured Media- an Experimental Study. SPEJ, 17, 1160-1171.

Alaskar, M., Ames, M., Horne, R., Li, K., Connor, S., and Cui, Y. (2010). In-situ Multifunction Nanosensors for Fractured Reservoir Characterization. Annual Meeting of the Geothermal Resources Council, Sacramento, California, 34.

Alaskar, M., Ames, M., Liu, C., Connor, S., Horne, R., Li, K., and Cui, Y. (2011). Smart Nanosensors for In-situ Temperature Measurement in Fractured Geothermal Reservoir. Annual Meeting of the Geothermal Resources Council, San Diego, California, 35 .

Alaskar, M., Li, K., and Horne, R. (2013). Influence of Particle Size on its Transport in Discrete Fractures: Pore-scale Visualization Using Micromodels. $38^{\text {th }}$ Stanford Geothermal Workshop, Stanford, California.

Alaskar, M., Li, K., and Horne, R. (2014). Transport of Temperature Nanosensors Through Fractured Tight Rock: an Experimental Study. SPE-SAS Annual Technical Symposium \& Exhibition, Al Khobar, Saudi Arabia.

Ames, M., Li, K., and Horne, R. (2013). The Utility of Threshold Reactive Tracers for Characterizing Temperature Distributions in Geothermal Reservoirs. Mathematical Geosciences, Special Issue.

Bamfield, P. (2001). Chromic Phenomena - Technological Applications of Colour Chemistry. Cambridge: The Royal Society of Chemistry.

Bancroft, C., Bowler, T., Bloom, B., and Clelland, C. T. (2001). Long-Term Storage of Information in DNA. Science, 293, 17631765.

Becker, M. W., Reimus, P. W., and Vilks, P (1999). Transport and Attenuation of Carboxylate-Modified Latex Microspheres in Fractured Rock Laboratory and Field Tracer Tests. Ground Water, 37(3), 387-395.

Brinker, C. J., and Scherer, G. W. (1990). Sol-Gel Science: The Physics and Chemistry of Sol-Gel Processing. San Diego: Academic Press Inc.

Chilkoti, A., Dreher, M. R., Meyer, D. E., and Raucher, D. (2002). Targeted Drug Delivery by Thermally Responsive Polymers. Adv. Drug Delivery Rev., 54, 613-630. 
Gringarten, A. C. and Sauty, J. P. (1975). A theoretical study of heat extraction from aquifers with uniform regional flow. Journal of Geophysical Research, 80(35), 4956-4962.

James, S. C. and Chrysikopoulos, C. V. (2003). Effective velocity and effective dispersion coefficient for finite-sized particles flowing in a uniform fracture. Journal of Colloid and Interface Science, 263, 288-295.

Keller, A. A., and Auset, M. A. (2007). Review of Visualization Techniques of Biocolloid Transport Processes at the Pore Scale Under Saturated and Unsaturated Conditions. Advanced Water Resour., 30, 1392-1407. doi:10.1016/j.advwatres.2006.05.013.

Kreft, A., and Zuber, A. (1978). On the physical meaning of the dispersion equation and its solutions for different initial and boundary conditions. Chemical Engineering Science., 33(11), 1471-1480.

Kreuter, J. (1994). Nanoparticles. In Kreuter, J. (Ed.), Colloidal Drug Delivery Systems, (pp. 219-342). Marcel Dekker. New York: NY, CRC press.

Moghimi, S. M., Hunter, A. C., and Murray, J. C. (2001). Long-Circulating and Target-Specific Nanoparticles: Theory to Practice. Pharmacol. Rev., 53, 283-318.

Panyam, J., and Labhasetwar, V. (2003). Biodegradable Nanoparticles for Drug and Gene Delivery to Cells and Tissue. Adv. Drug Del. Rev., 55, 329-347.

Panyam, J., Sahoo, S. K., Prabha, S., Bargar, T., and Labhasetwar, V. (2003). Fluorescence and Electron Microscopy Probes for Cellular and Tissue Uptake of poly(D,L-lactide-coglycolide) Nanoparticles. Int. J. Pharm., 262, 1-11.

Paunescu, D., Fuhrer, R., and Grass, R. (2013). Protection and Deprotection of DNA - High-Temperature Stability of Nucleic Acid Barcodes for Polymer Labeling. Angew. Chem. Int. Ed., 52, 4269-4272.

Popping, B. (2002). The Application of Biotechnological Methods in Authenticity Testing. J. Biotechnol., 98, 107-112.

Rahman, I. A., Vejayakumaran, P., Sipaut, C. S., Ismail, J. (2007). An optimized Sol-gel Synthesis of Stable Primary Equivalent Silica Particles. Colloids and Surfaces A: Physicochem. Eng. Aspects, 294, 102-110.

Redden, G., Stone, M., Wright, K. E., Mattson, E., Palmer, C. D., Rollins, H., Harrup, M., and Hull, L. C. (2010). Tracers for Characterizing Enhanced Geothermal Systems. Proceedings of Thirty-Fifth Workshop on Geothermal Reservoir Engineering, Stanford University, Stanford, CA.

Reimus, P. W. (1995). The Use of Synthetic Colloids in Tracer Transport Experiments in Saturated Rock Fractures. PhD Thesis. University of New Mexico.

Rose, P., Riasetto, D., Siy, J., Bartl, M., Reimus, P., Mella, M., Leecaster, K., and Petty, S. (2011). Quantum Dots as Tracers in Geothermal and EGS Reservoirs. Proceedings of Thirty-Sixth Workshop on Geothermal Reservoir Engineering, Stanford University, Stanford, CA.

Saggaf, M.M. (2008). A Vision for Future Upstream Technologies. Journal of Petroleum Technology, 60, 3. 
Alaskar et al.

Saleh, S. M., Muller, R., Mader, H. S., Duerkop, A., and Wolfbeis, O. S. (2010). Novel Multicolor Fluorescently Labeled Silica Nanoparticles for Interface Fluorescence Resonance Energy Transfer to and from Labeled Avidi. Anal. Bioanal. Chem., 398, $1615-1623$.

Seeboth, A., Klukowska, A., Ruhmann, R., and Lotzsch, D. (2007). Thermochromic Polymer Materials. Chin. J. Polym. Sci., 25(2), 123-135.

Sutton, D., Nasongkla, N., Blanco, E., and Gao, J. (2007). Functionalized Micellar Systems for Cancer Targeted Drug Delivery. Pharmaceutical Research, 24.(6), 1029-1046.

Wan, J. M., and Wilson, J. L. (1994a). Of the Role of the Gas-Water Interface on the Fate and Transport of Biocolloids in PorousMedia. Water Resources Res., 30,11-23.

Wan, J. M., and Wilson, J. L. (1994b).Colloid Transport in Unsaturated Porous Media. Water Resources Res., 30, 857-64.

White, M. A. and LeBlanc, M. (1999). Thermochromism in Commercial Products. J. of Chem. Education, 76 (9).

Williams, M. D., D. Newell, V. R. Vermeul, T. Watson, and P. W. Reimus (2010). "Development of Models to Simulate Tracer Behavior in Enhanced Geothermal Systems - Status Report,” Pacific Northwest National Laboratory, PNNL-19523.

Wu, E. C., Park, J. H., Park, J., Segal, E., Cunin, F., and Sailor, M. J. (2008). Oxidation-Triggered Release of Fluorescent Molecules or Drugs Form Mesoporous Si Microparticles. ACS Nano, 2 (11), 2401-2409. 
Table 1: Summary of physical parameters used in analytical model

\begin{tabular}{|c|c|c|}
\hline Parameter & Value & Units \\
\hline$L$ & 1000 & $m$ \\
\hline$W$ & 1000 & $m$ \\
\hline$b$ & 1 & $\mathrm{~mm}$ \\
\hline$T_{0}$ & 200 & ${ }^{\circ} \mathrm{C}$ \\
\hline$T_{I N J}$ & 100 & ${ }^{\circ} \mathrm{C}$ \\
\hline$\rho_{W}$ & 865 & $\frac{\mathrm{kg}}{\mathrm{m}^{3}}$ \\
\hline$C_{W}$ & 4491 & $\frac{J}{k^{\circ} \mathrm{C}}$ \\
\hline$\rho_{R}$ & 2600 & $\frac{\mathrm{kg}}{\mathrm{m}^{3}}$ \\
\hline$C_{R}$ & 816 & $\frac{J}{k g^{\circ} \mathrm{C}}$ \\
\hline$K_{R}$ & 2 & $\frac{W}{m^{\circ} \mathrm{C}}$ \\
\hline$q$ & 0.02 & $\frac{m^{3}}{s}$ \\
\hline$D_{\text {Taylor, sol }}$ & 0.019 & $\frac{m^{2}}{s}$ \\
\hline$D_{\text {Taylor, }}{ } P$ & 0.022 (for $100 \mathrm{~nm}$ particle) & $\frac{m^{2}}{s}$ \\
\hline$e$ & $10 \% *(2 b)$ & $\mathrm{mm}$ \\
\hline$A$ & $2.5 \times 10^{7}$ & $\frac{1}{S}$ \\
\hline$E_{A}$ & 113 & $\frac{\mathrm{kJ}}{\mathrm{mol}}$ \\
\hline
\end{tabular}


Alaskar et al.

Table 2: Summary of parameter variation in sensitivity analysis

\begin{tabular}{cccc}
\hline Case & $t_{\text {OP }}[y r]$ & $\left.T_{\text {THRESH }}{ }^{\circ} \mathrm{C}\right]$ & $\begin{array}{c}\sigma_{\text {NOISE }}[\% \text { of } \\
\text { maximum signal] }\end{array}$ \\
\hline Base Case -1 & 1 & 199 & 3 \\
2 & 2 & 199 & 3 \\
3 & 3 & 199 & 3 \\
4 & 1 & 190 & 3 \\
5 & 1 & 150 & 6 \\
\hline
\end{tabular}

Table 3: Summary of the physical properties of the glass beads packed column ${ }^{\text {a }}$

\begin{tabular}{|c|c|c|c|c|}
\hline $\bar{d}$ & $\bar{L}$ & $P V$ & $\emptyset_{\text {sat }}$ & $k_{w}$ \\
\hline$(\mathrm{cm})$ & $(\mathrm{cm})$ & $\left(\mathrm{cm}^{3}\right)$ & (\%) & (darcy) \\
\hline 1.20 & 41.50 & 21.54 & 35.90 & 5.23 \\
\hline \multicolumn{5}{|c|}{${ }^{a} d$ and L are column diameter and length, respectively, $\emptyset_{\text {sat }}$ is porosity by resaturation, $k_{w}$ is the water } \\
\hline
\end{tabular}




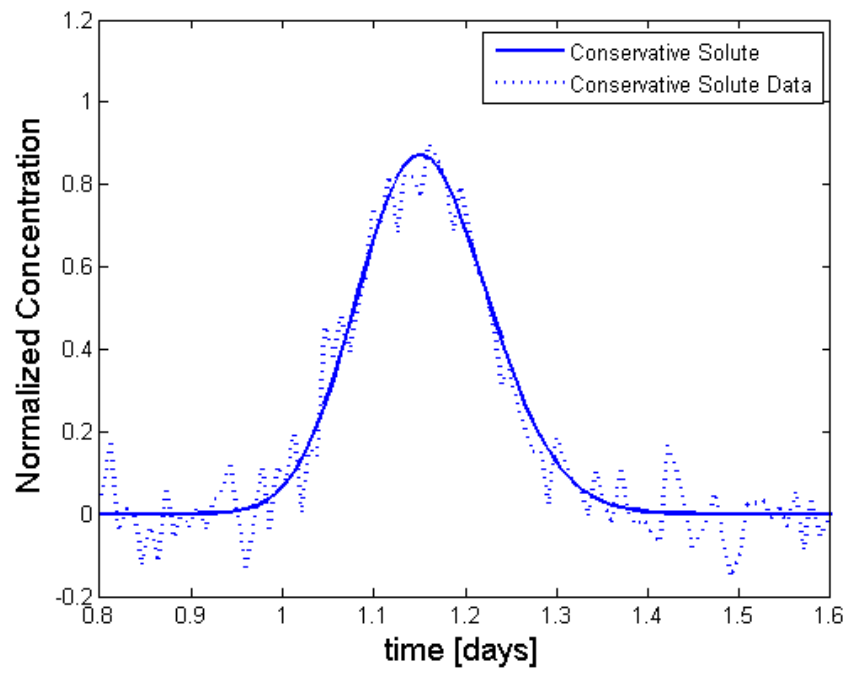

Figure 1: Return curve for conservative solute tracer showing synthetic data and fit model.

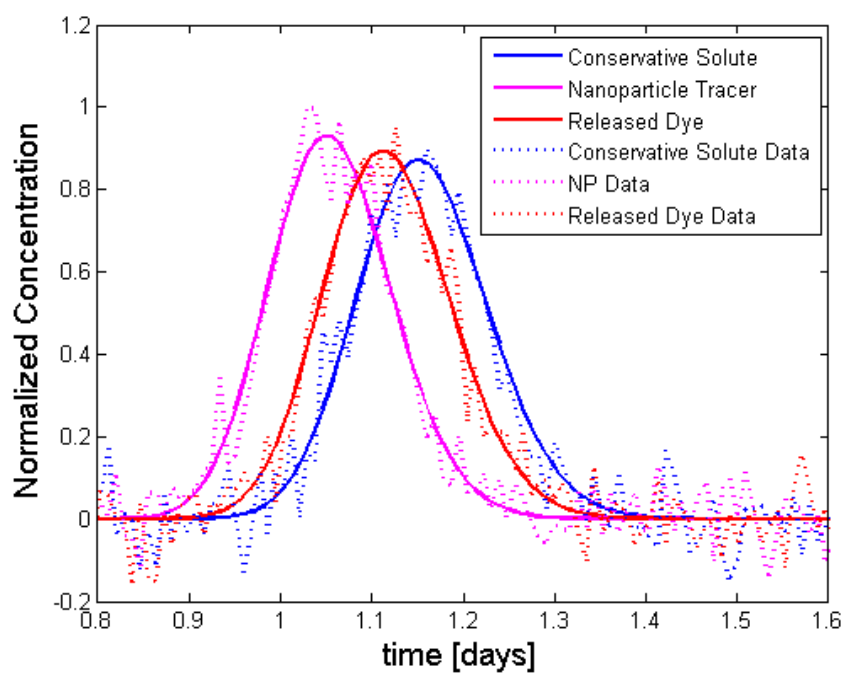

Figure 2: Return curves for dye-releasing nanotracer showing synthetic data and fit model. 
Alaskar et al.

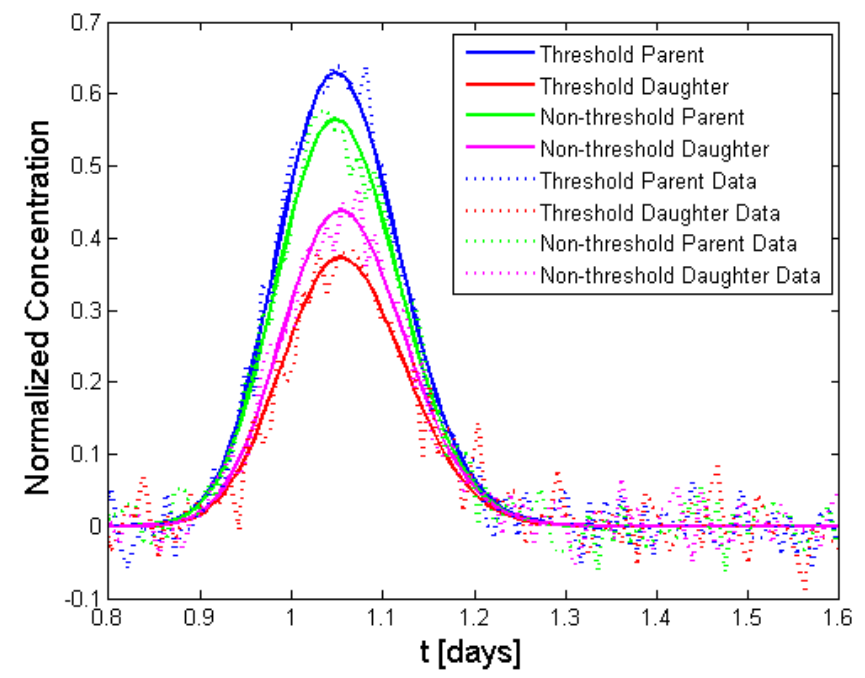

Figure 3: Return curves for nanoreactor showing synthetic data and fit model.
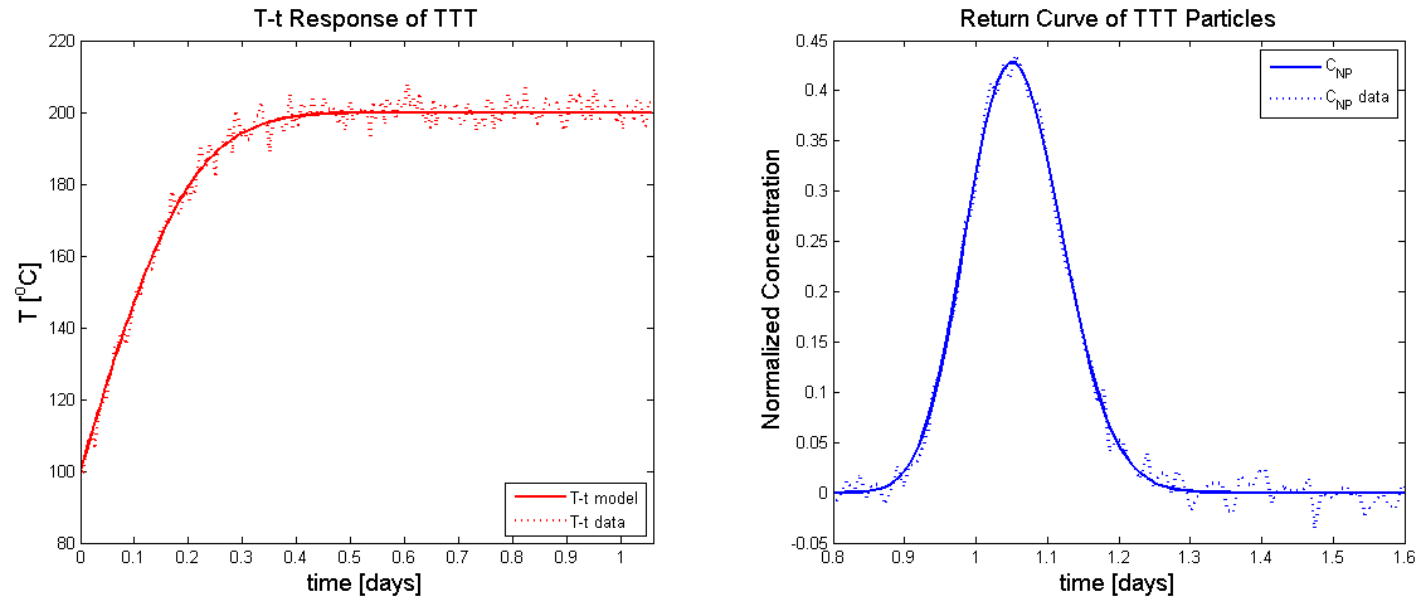

Figure 4: Temperature-time response for average-velocity TTT particle and TTT nanoparticle return curves showing synthetic data and fit model. 

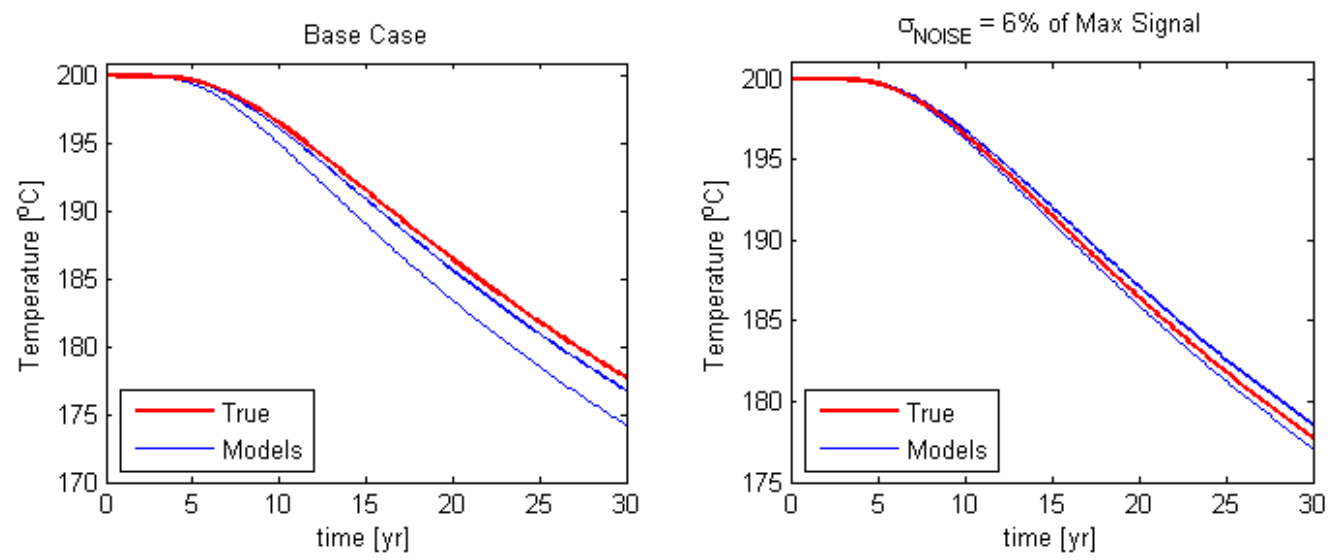

Figure 5: Thermal breakthrough curves from 1000 solutions to inverse problem for conservative solute tracer.
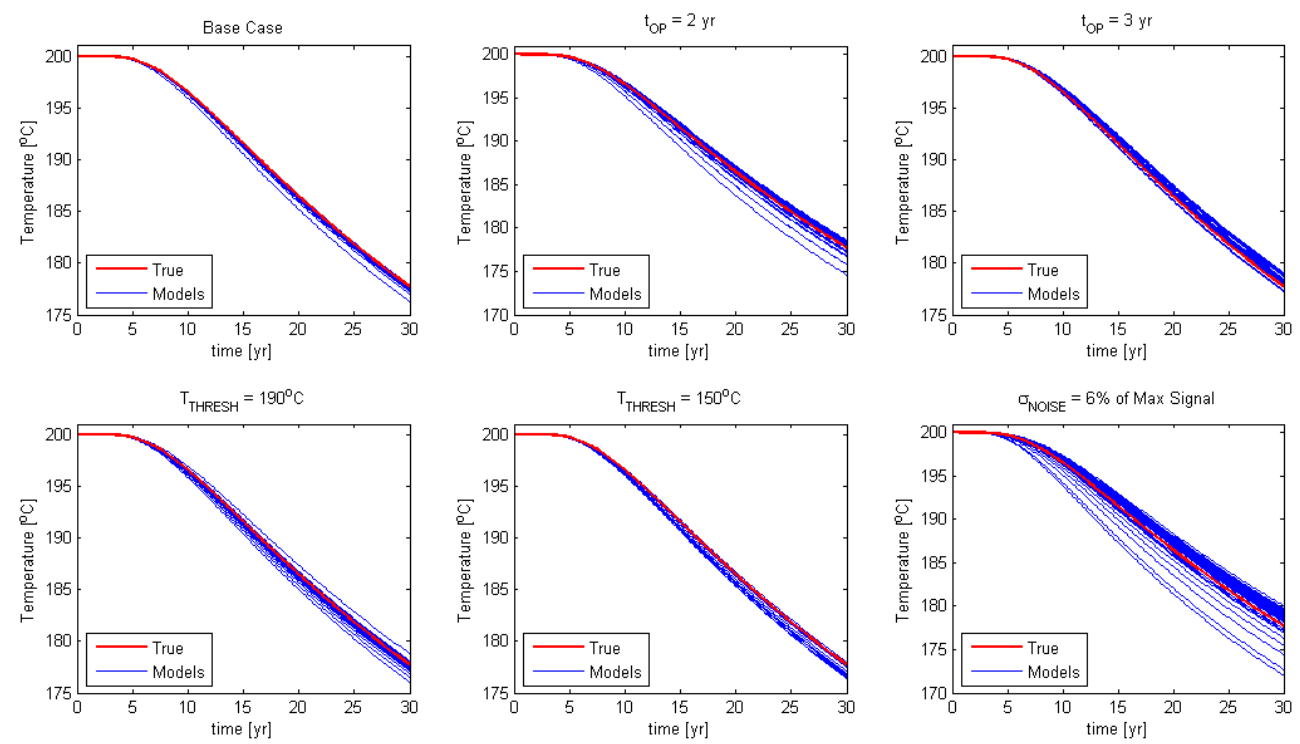

Figure 6: Thermal breakthrough curves from 1000 solutions to inverse problem for dye-releasing nanotracer. 

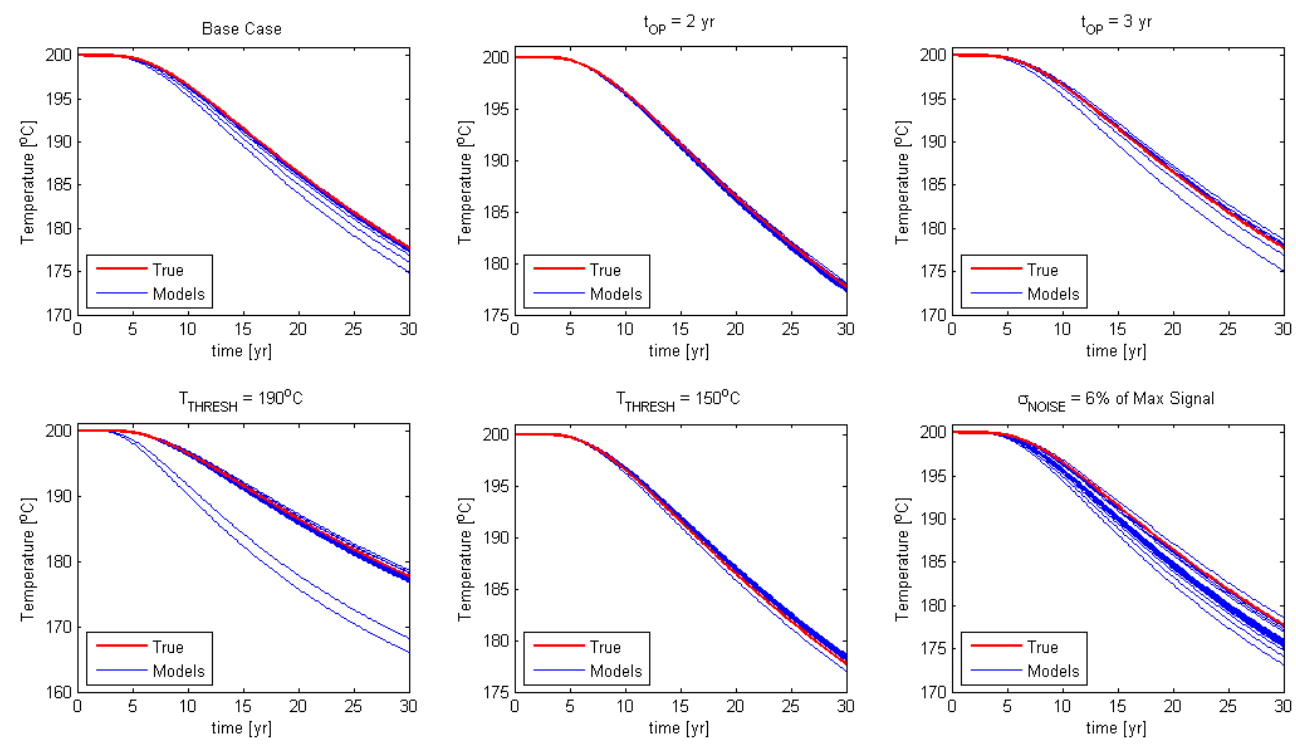

Figure 7: Thermal breakthrough curves from 1000 solutions to inverse problem for threshold nanoreactor.
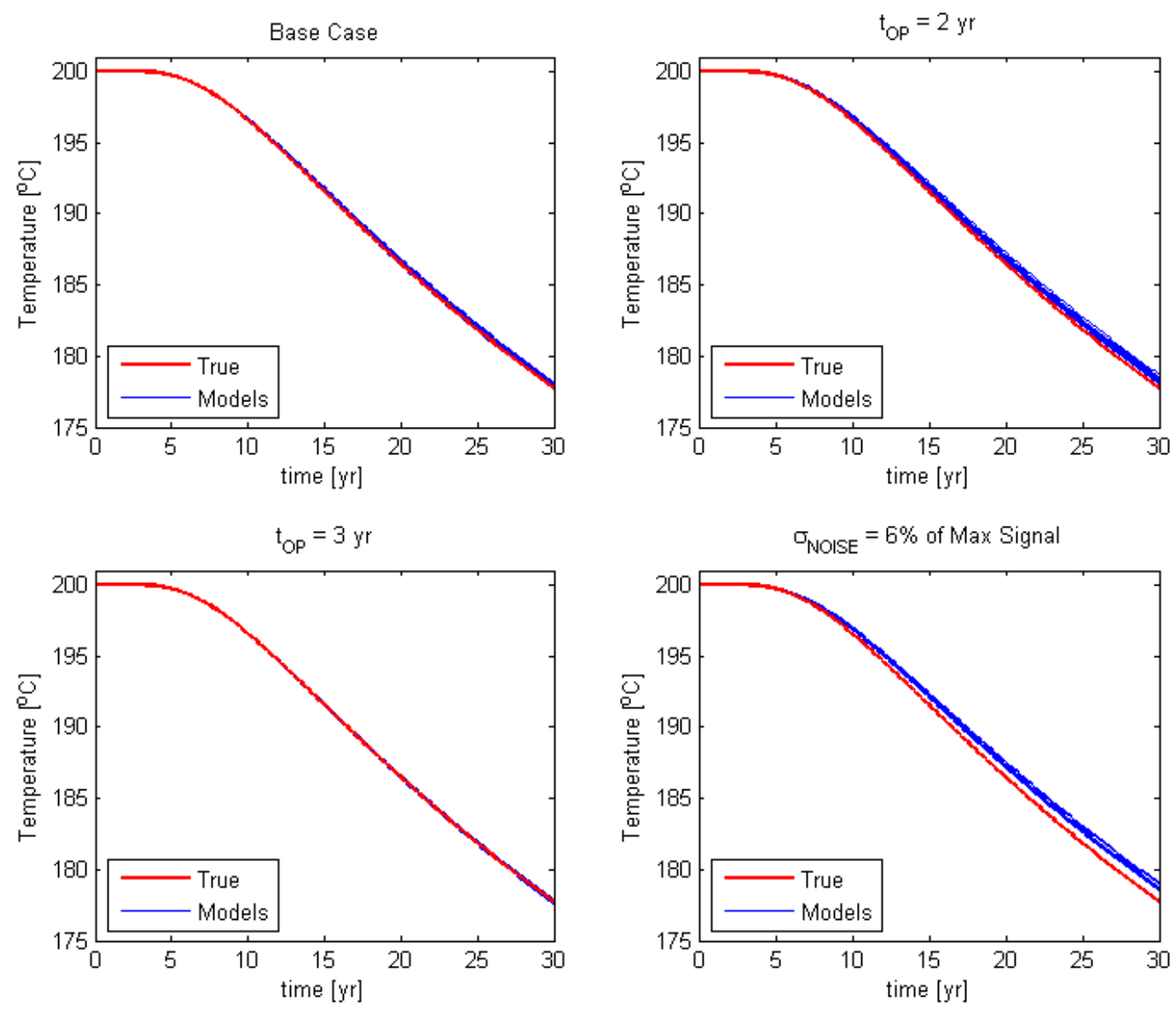

Figure 8: Thermal breakthrough curves from 1000 solutions to inverse problem for TTT. 
A
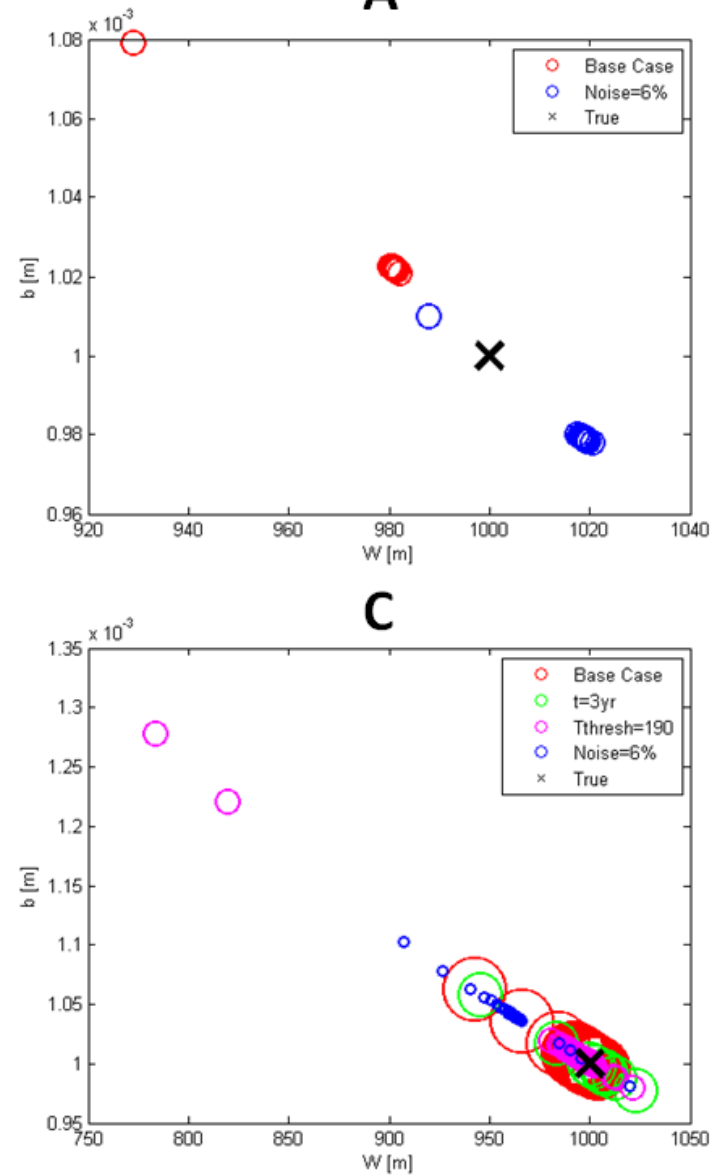

B

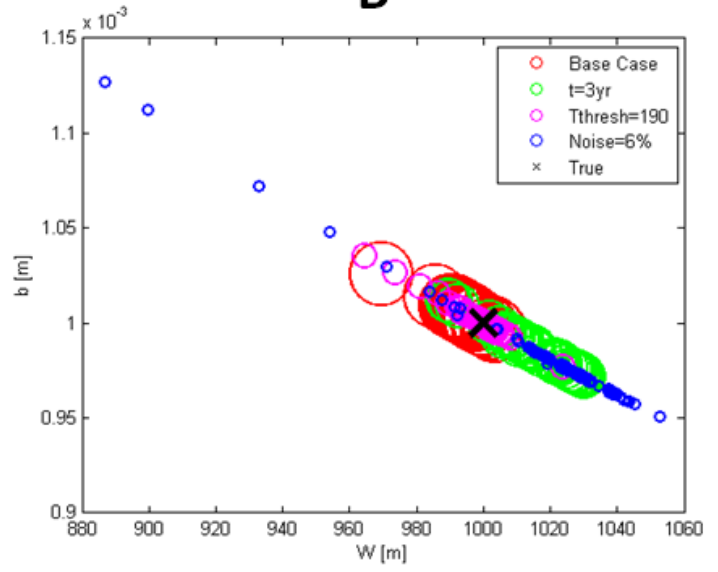

D

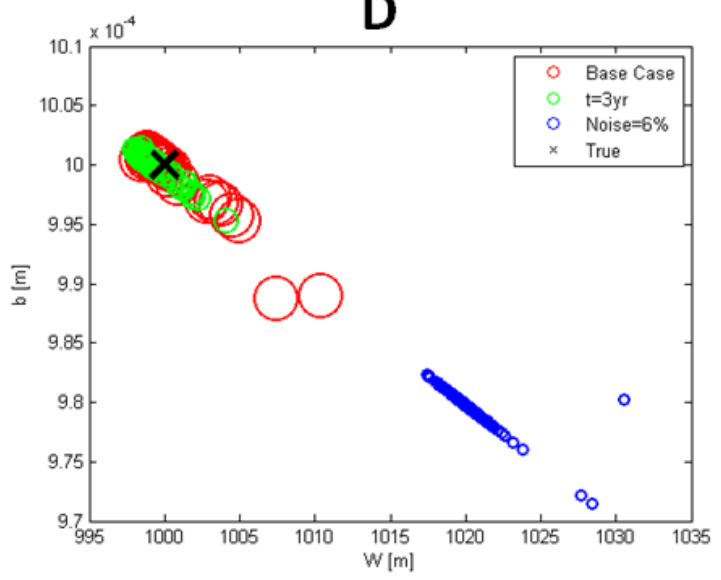

Figure 9: Scatter plots of decision variables from 1000 solutions to inverse problem for (A) conservative solute tracer, (B) dye-releasing nanotracer, (C) nanoreactor, and (D) TTT.

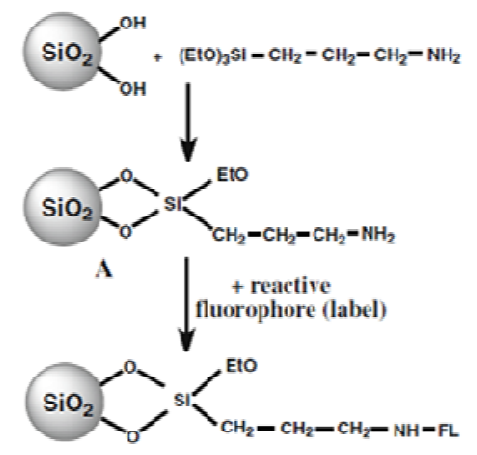

B

Figure 10: Schematic representation of silica nanoparticle surface modification and dye attachment. Adopted from Saleh et al. (2010). 


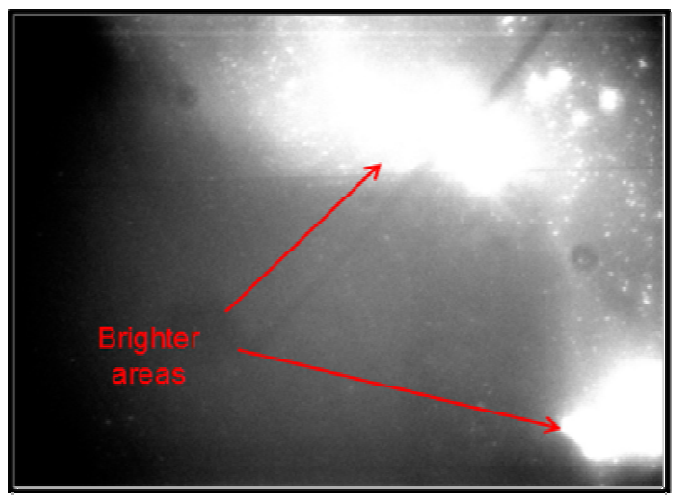

Figure 11: Fluorescent microscopy image of dye-attached silica nanoparticles on quartz substrate. The whole substrate was bright (compared to black). Brighter areas indicated that there were more fluorescent molecules at these locations.
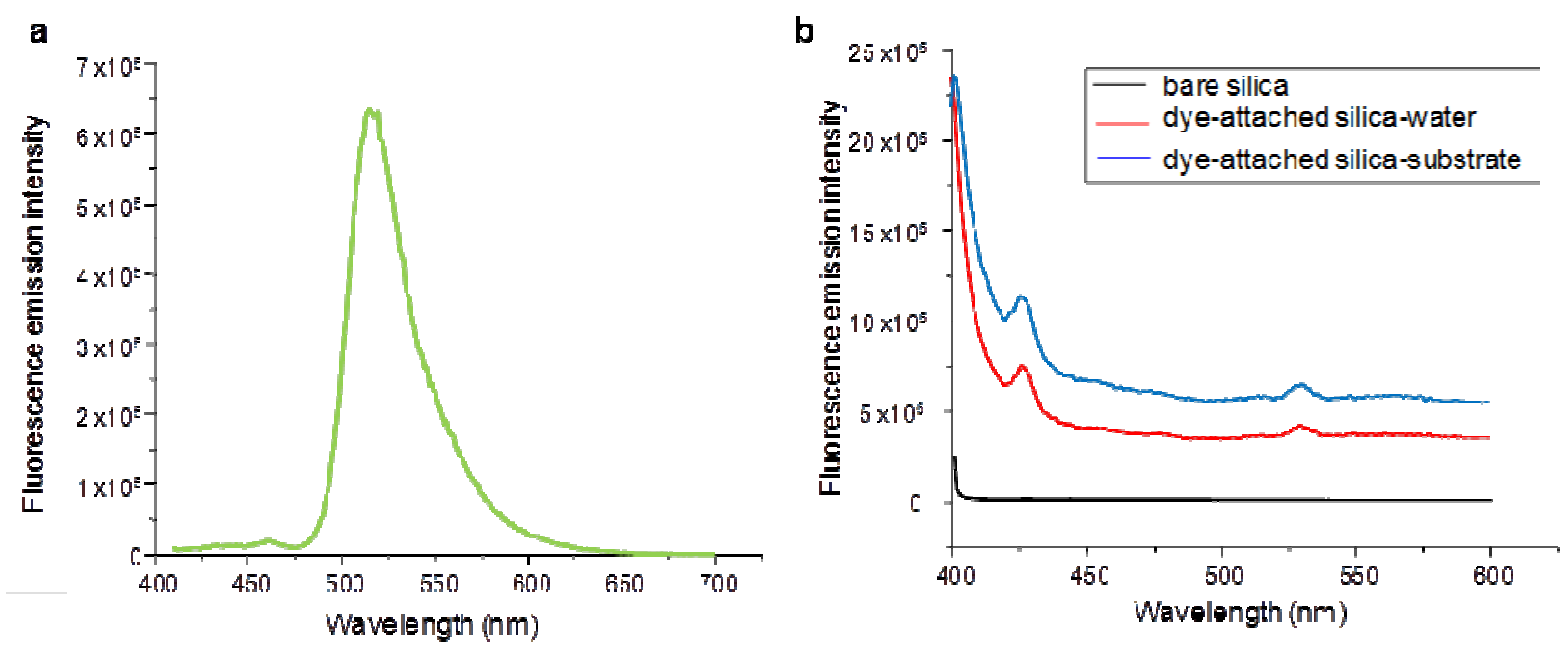

Figure 12: Fluorescence spectrum of (a) Oregon 488 dye molecule solution and (b) bare silica (no dye attachment) nanoparticles as control (black), dye-attached silica nanoparticles in water (red) and dye-attached silica nanoparticles on quartz substrate (blue). All samples were excited at wavelength of $400 \mathrm{~nm}$. The dye-attached silica samples showed two peaks at 425 and $530 \mathrm{~nm}$, compared to the free dye (Oregon 488 ) that has one peak at $530 \mathrm{~nm}$. 


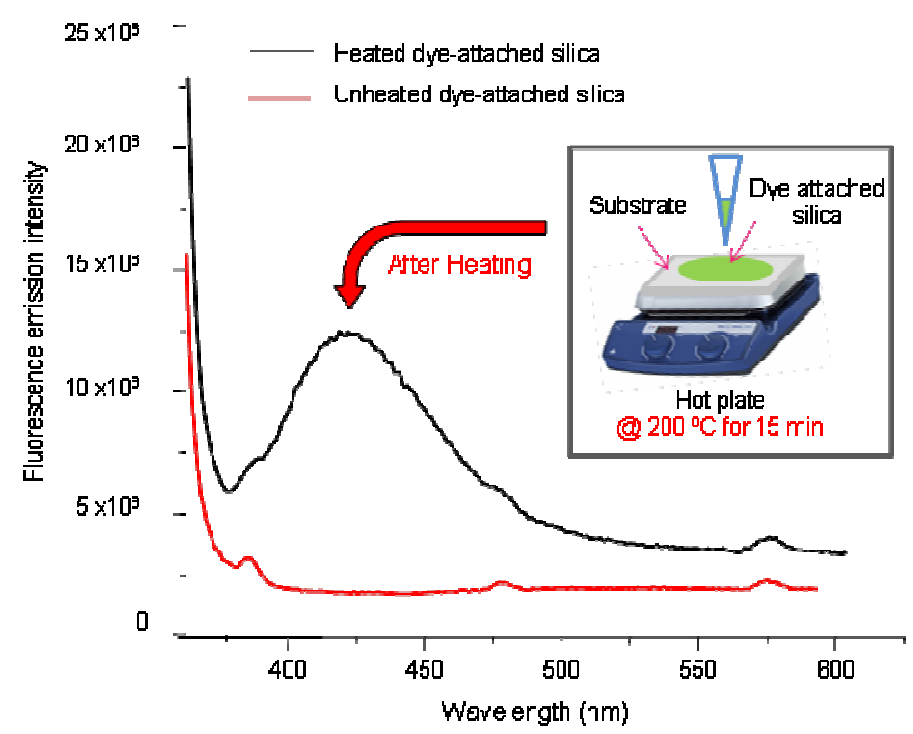

Figure 13: Fluorescence spectrum of the dye-attached silica nanoparticle before (red) and after (black) heating at $200^{\circ} \mathrm{C}$ for $15 \mathrm{~min}$. The samples were excited at wavelength of $360 \mathrm{~nm}$. The change in the fluorescent spectrum can be readily distinguished as an indication of a temperature exposure.

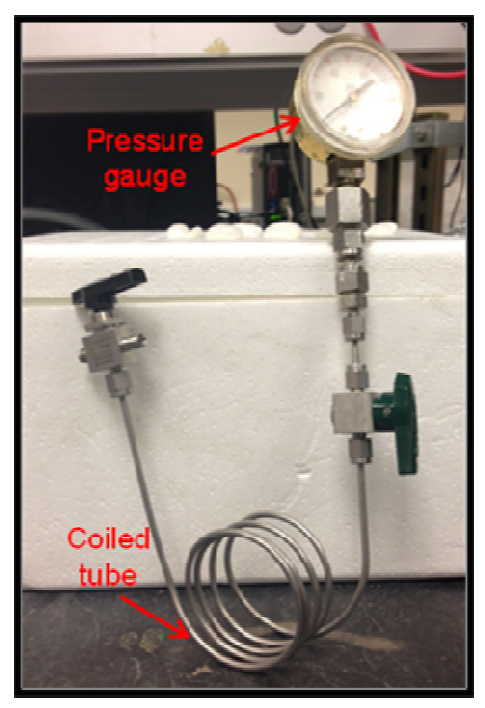

Figure 14: A picture of the coiled tube used during the bench heat test of the irreversible thermochromic ink. The coiled tube is submerged in oil bath heated to desired temperature for three minutes. 


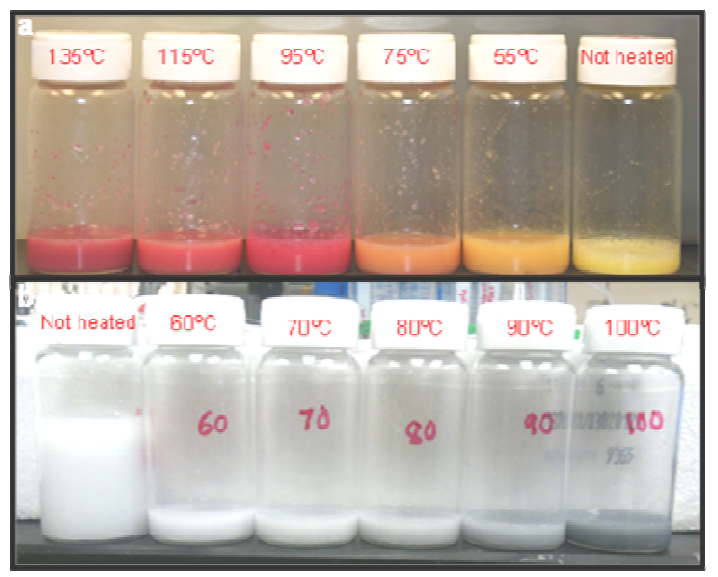

Figure 15: Snapshots of (a) black and (b) magenta irreversible thermochromic ink samples heated for three minutes at different temperatures below and above their reaction temperature. The density or intensity of the sample color is increasing with increasing temperature. The color is fully developed at about $35^{\circ} \mathrm{C}$ and $10^{\circ} \mathrm{C}$ above the rated temperature of the black and magenta concentrate samples, respectively.

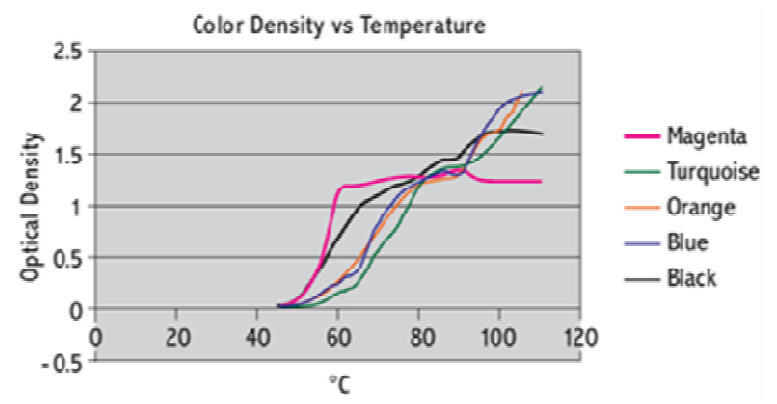

Figure 16: Color density as function of temperature of various irreversible thermochromic inks heated for three minutes.

This test was performed by the manufacturer (Adopted from the product color comparison chart, LCR Hallcrest LLC).

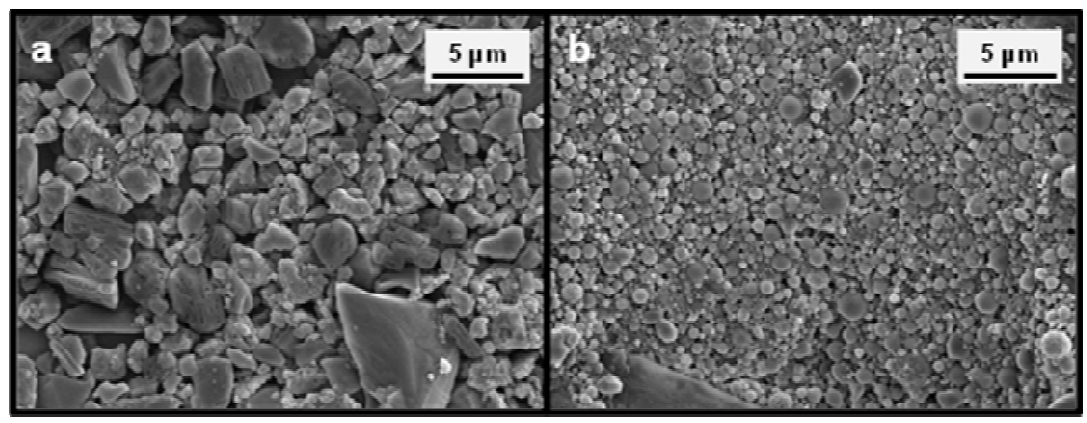

Figure 17: Electron micrographs of the black thermochromic concentrate (a) before and (b) after heating. By comparison, it can be clearly seen that the particles change their structures after heating to form spherical particles. 


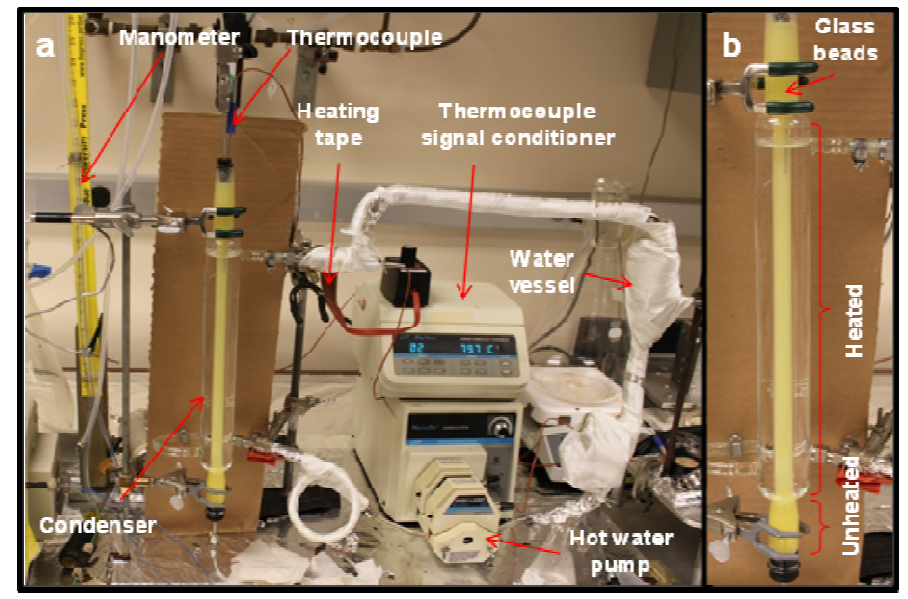

Figure 18: (a) Experimental apparatus used during the combined heat and transport experiment, and (b) close-up image of a condenser column packed with glass beads.

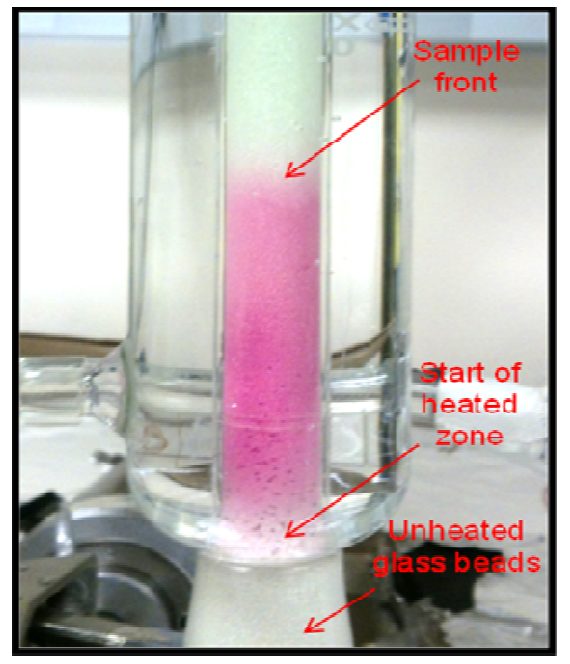

Figure 19: A snapshot of the thermochromic sample entering the heated zone of the glass beads column. Flow is upwards from the bottom. The sample has changed its color immediately from off-white to magenta as a result of temperature. The change in color confirmed that the column temperature is at least $80^{\circ} \mathrm{C}$. 


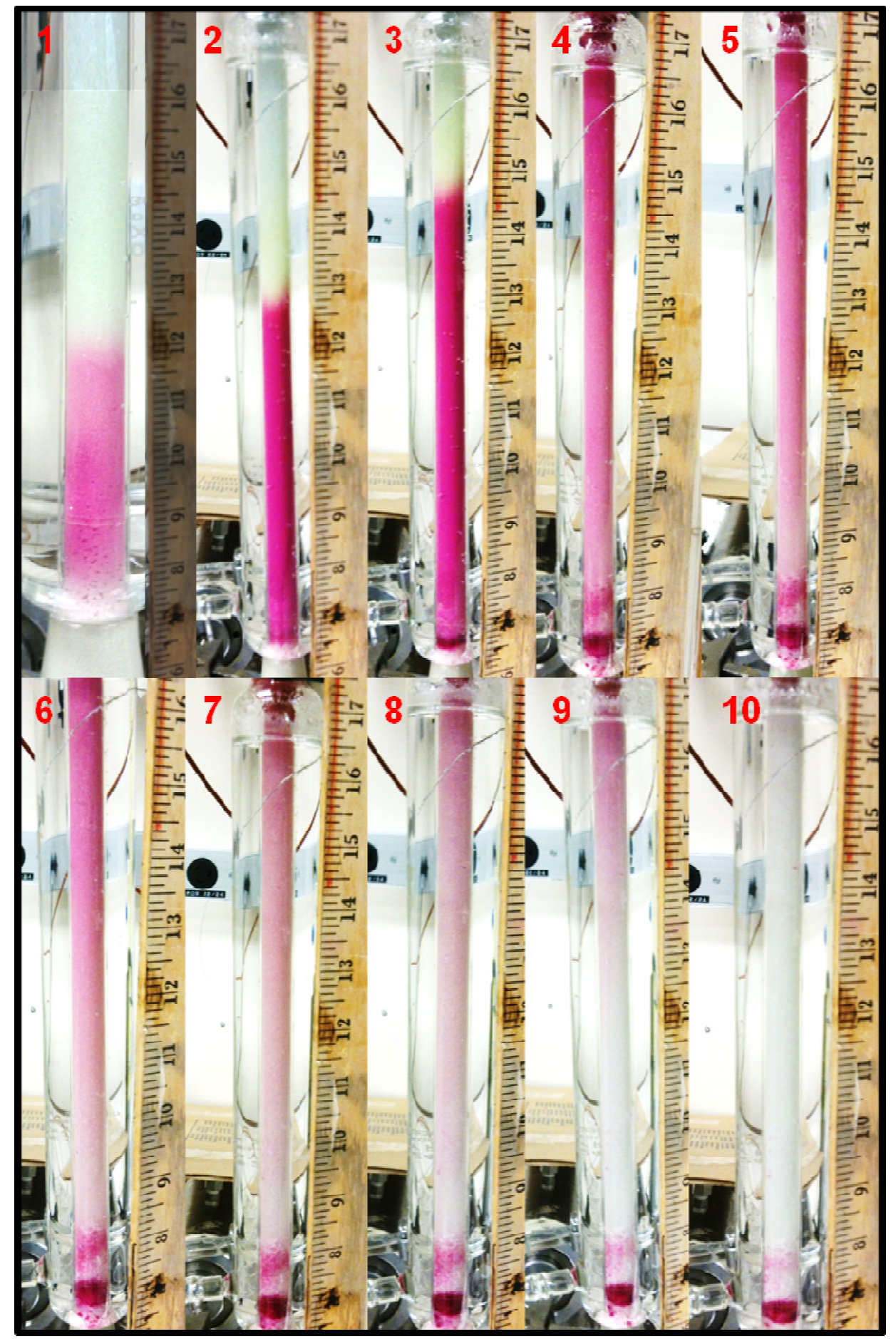

Figure 20: Snapshots of the thermochromic slug during its flow under heat through the glass bead column. Flow is upwards. 


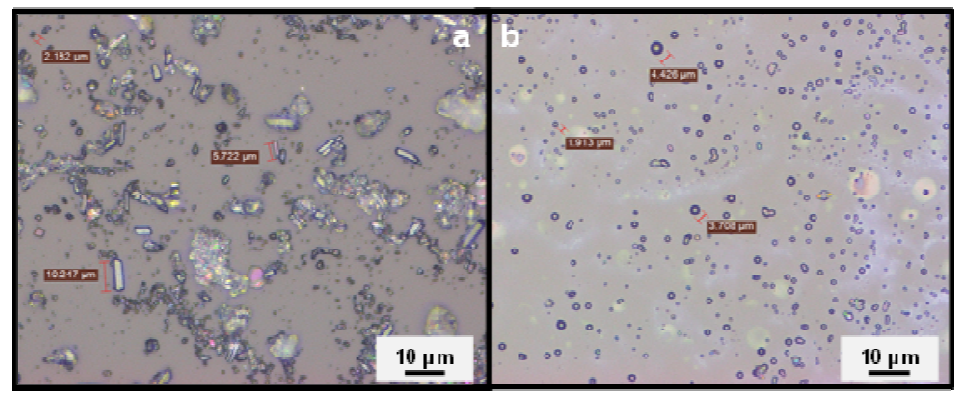

Figure 21: Optical images of the (a) unheated and (b) heated thermochromic sample. The particles change shape from crystalline to spherical solids.

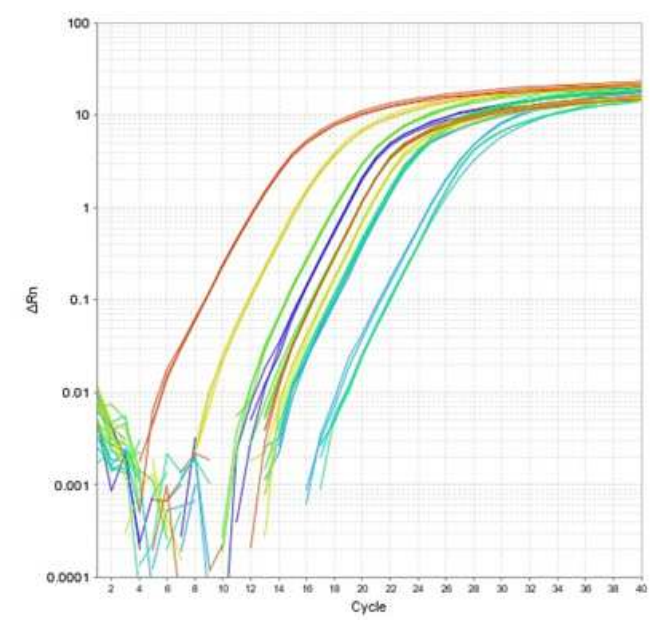

Figure 22: Amplification plot of standards and nucleic acid samples.

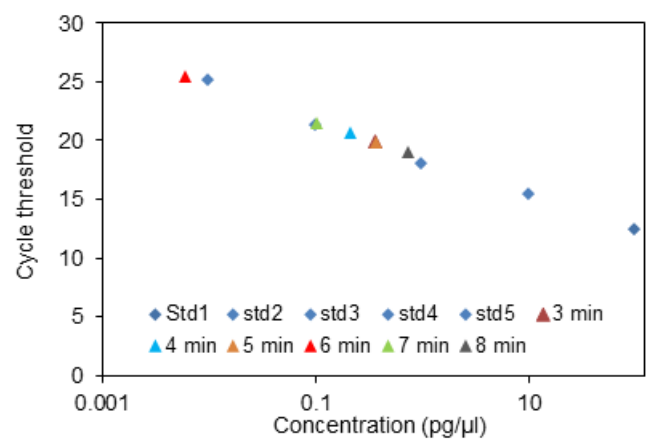

Figure 23: Standard curve of standards and samples showing the relation between the cycle threshold and DNA concentration (DNA concentration in picogram per microliter). 


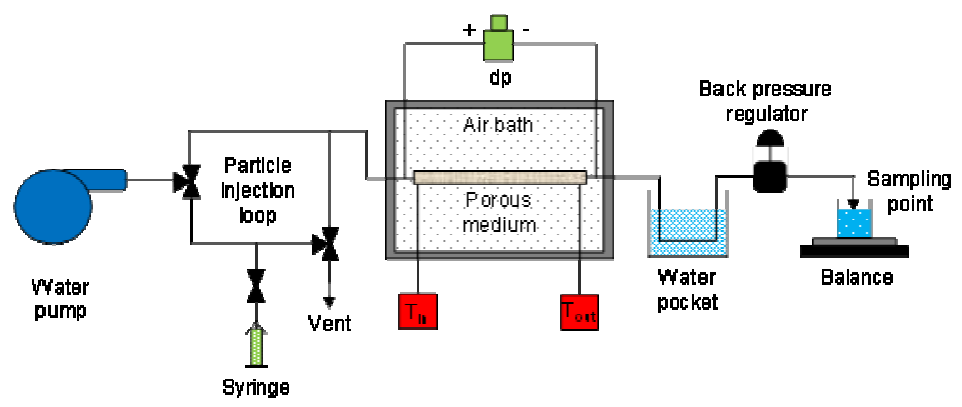

Figure 24: Schematic of the experimental apparatus for testing the silica-encapsulated DNA particles.
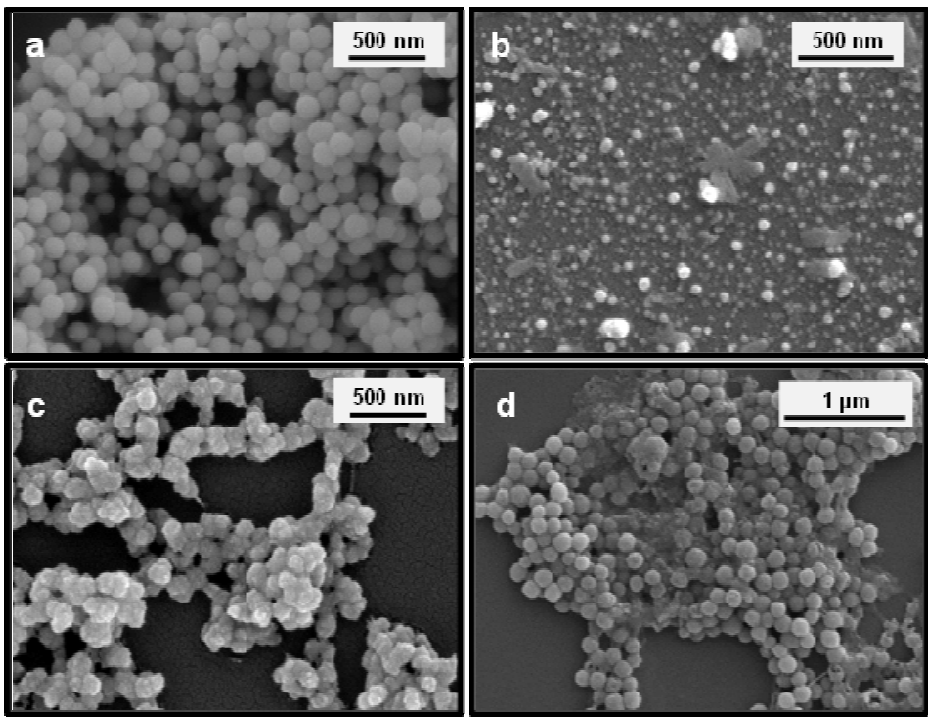

Figure 25: Electron micrograph of (a) influent, (b) and (c) effluent samples during particle injection at $150^{\circ} \mathrm{C}$ showing reduction in particle size and particle aggregation, respectively, (d) effluent sample collected during injection at room temperature where neither the particle size reduction nor aggregation of particles were observed. 